\title{
Dynamic nonlinear connectedness between the green bonds, clean energy, and stock price: the impact of the COVID-19 pandemic
}

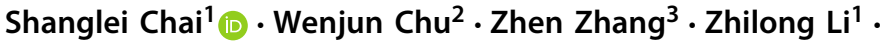 \\ Mohammad Zoynul Abedin ${ }^{4,5}$
}

Accepted: 16 November 2021

(c) The Author(s), under exclusive licence to Springer Science+Business Media, LLC, part of Springer Nature 2021

\begin{abstract}
This paper uses weekly data from July 01, 2011 to July 09, 2021 to examine the dynamic nonlinear connectedness between the green bonds, clean energy, and stock price around the COVID-19 outbreak in the global markets. By building a time-varying parameter vector autoregression model (TVP-VAR), the comparison analyses of pre- and during the COVID19 sample groups verify the existence of nonlinear and dynamic correlation among the three variables. First, prior to the COVID-19 pandemic, the simultaneous impacts of clean energy on stock price increased over time. Second, the results of impulse responses at different horizons indicate that green bonds lead to a short-term increase of clean energy, and it exerts an increasingly positive impacts after the COVID-19 outbreak. The COVID-19 has weakened the negative impacts of green bonds on stock price in the medium term. Finally, through the analysis of impulse responses at different points, we find that stock prices will rise when clean energy is subjected to a positive shock, and this positive effect is stronger during economic recovery period than in the other two periods.
\end{abstract}

Keywords Green bonds · Clean energy market · Stock price · COVID-19 pandemic . TVP-VAR

$凶 \quad$ Mohammad Zoynul Abedin abedinmz@yahoo.com

1 Business School, Shandong Normal University, Jinan 250014, China

2 School of Economics and Management, China University of Petroleum, 66 West Changiiang Road, Huangdao District, Qingdao 266555, China

3 Institute of Systems Engineering, Dalian University of Technology, Dalian 116024, China

4 Department of Finance, Performance \& Marketing, Teesside University International Business School, Teesside University, Middlesbrough, Tees Valley TS1 3BX, UK

5 Department of Finance and Banking, Hajee Mohammad Danesh Science and Technology University, Dinajpur 5200, Bangladesh 


\section{Introduction}

As one of the most destructive global events, COVID-19 poses serious challenges to the global economy, society, and environment. With the increase in the unemployed population and the sharp decline in production and consumption, the economy has taken a hard hit (Chen, 2020). The breakout of the COVID-19 pandemic has brought major setbacks to economic growth (Abu-Rayash \& Dincer, 2020; Tisdell, 2020). Azomahou et al. (2021) confirm that COVID-19 has caused the forecasted GDP growth loss in Africa. Although the COVID-19 pandemic has destroyed the global economy, it has also led to an increase in the sharing of clean and renewable energy and a decrease in fossil fuels (Halbrügge et al., 2021; Wan et al., 2021), resulting in the demand for green bonds. Halbrügge et al. (2021) conclude that the COVID-19 epidemic has led to a decline in electricity demand and an increase in the proportion of renewable energy consumption. Yi et al. (2021) consider that COVID-19 pandemic has significantly influenced the green bond market. Mensi et al. (2021) document that COVID-19 may lead to the inefficiency of green bond market. However, Naeem et al. (2021) confirm that green bond markets are more efficiency during the COVID-19 pandemic. In addition to the effects on the green bonds and clean energy mentioned above, COVID19 also affects stock markets (Xu, 2021). Ashraf (2021) verifies the negative impact of the COVID-19 epidemic on stock returns. Ftiti et al. (2021) find that the shock of COVID-19 increases the volatility of stock returns and the liquidity risks. Liu, Wei, et al. (2021) indicate that the COVID-19 epidemic enhances the risk contagion effect of the stock market. With the international popularity of the green economy concept in recent decades, the impact of the COVID-19 epidemic on the relationship between green bonds, clean energy, and stock prices has become increasingly prominent.

A number of recent studies has highlighted the connectedness between green bonds, clean energy and stock price. Figure 1 presents the literature focused on the relationship between three variables. Green bonds play a crucial role in renewable and clean energy projects (European Commission, 2016). Thus, there is a strong link between green bonds and clean energy. On the one hand, there exists a one-way connectedness between clean energy and green bonds. McInerney and Bunn (2019) conclude that the issuance of green bonds contributes to the development of clean energy, such as bioenergy. Kung et al. (2022) provide evidence that green bonds positively impact bioenergy. On the other hand, the bidirectional relationship is also considered (Liu, Liu, et al., 2021; Pham et al., 2021; Ferrer et al., 2021; Nguyen et al., 2021). The connectedness between green bonds and stock prices is also investigated. Some literature has considered the connectedness and co-movement between green bonds and stock price, however, the connectedness and co-movement are limited (Ferrer et al., 2021; Reboredo, 2018; Reboredo \& Ugolini, 2020). Besides, Wang et al. (2020) find that green bonds can positively influence stock returns. Another strand of the literature also investigates the risk spillover (Gao et al., 2021; Kuang, 2021). Gao et al. (2021) demonstrate that green bond market is affected by one-way risk spillovers from the stock market. What's more, stock prices are also closely related to clean energy. Paramati et al. (2016) and McInerney and Bunn (2019) confirm that the development of stock market contributes to clean energy market. Lundgren et al. (2018) argue that European stocks are heavily dependent on clean energy stock prices. Recent studies have emerged that highlight the positive relationship and co-movement between stock price and clean energy (Elsayed et al., 2020; Razmi et al., 2020). However, the outbreak of COVID-19 may lead to a structural change in the connectedness between green bonds, clean energy and stock prices. The COVID-19 pandemic has triggered the global economic crisis (Huang \& Liu, 2021), resulting in extreme market conditions. 


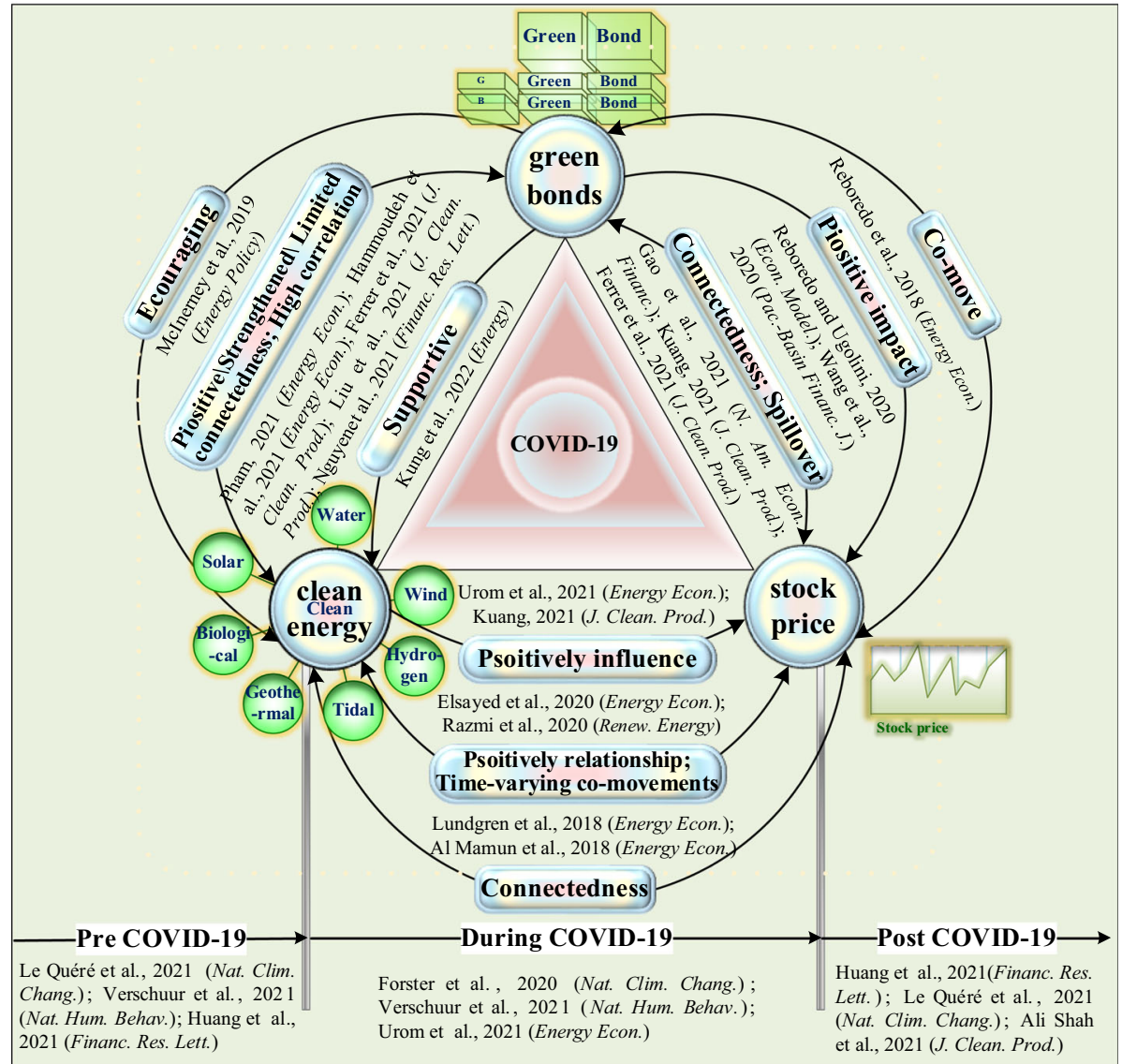

Fig. 1 The relationship between the green bonds, clean energy and stock price

Pham (2021) find that the connectedness between green bonds and clean energy has been enhanced during the extreme market conditions. What's more, Kuang (2021) demonstrates green bonds have evidently reduced the tail risk of equity indices during the COVID-19. However, rare literature focused on the shock of COVID-19 pandemic on the connectedness between variables. Therefore, we examine the effects of the COVID-19 pandemic on the connectedness between green bonds, clean energy and stock prices.

The theoretical analysis of the connectedness between green bonds, clean energy, and stock price may be as follows. Environment, Social Responsibility, Corporate Governance (ESG) has gradually become the core of enterprises' development. Environmentally friendly enterprises, such as clean energy companies, can obtain funds through issuing green bonds. The development of large scale clean energy projects also requires significant investment (Lei et al., 2020). And green bonds can support the development of clean energy projects, which can influence clean energy market. What's more, enterprises that cannot transit to clean energy, such as coal mines or steel companies, will find it increasingly difficult to sell bonds. Therefore, there exists a closely relationship between green bonds and clean energy. From the perspective of investors, the individual behavior of investors sometimes leads to a 
linkage response between the green bond market and stock market. Many individual investors will participate in the green bond and stock markets at the same time. Investors have certain expectations about risks. When the risk of a market exceeds their risk expectations, they may transfer from this market to other markets. Moreover, investors' funds are limited. If investors encounter a major loss in one market, it is likely to cause the investors to monetize investment in another market and re-invest in the portfolio. However, not every investor has a complete investment knowledge system. Most investors may change the amount of investment due to fear that another market is also depressed when one market is down. From the perspective of the enterprises, enterprises' issuance of green bonds may encourage enterprises to enlarge production, which will contribute to the performance of enterprises in the stock market. With the development of green economy, clean energy enterprises perform well in the stock market, which will promote stock price. And the performance of stock market will influence the enterprises' financing capability, thus impacting enterprises' production which plays a vital role in clean energy consumption and investment.

In this context, we investigate the connectedness between the green bonds, clean energy, and stock price. This paper contributes to the extant literature in three ways: (1) We use weekly data spanning from July 01, 2011 to July 09, 2021, covering the period of the COVID-19 pandemic. An important point that has been ignored in previous studies is the lack of considering different economic conditions while examining the relationship between economic variables. The TVP-VAR model can interpret the effect of variables shock in different economic conditions. Since the global economy always volatile, we intend to shed light on the dynamic connectedness between green bonds, clean energy, and stock price. And we also consider the shock of COVID-19 pandemic on the connectedness between three variables; (2) Impulse response analysis at different time horizons and time points investigates the effects of variables from the perspectives of short term, medium term, and long term to more clearly depict the influence and duration of the interaction between variables; (3) It proposes important conclusions and suggestions. Although green bonds may restrain clean energy in the medium term, it can promote stock price in the short and long term. The development of clean energy may hinder the green bonds and stock price in the medium term. Furthermore, the negative impact of clean energy on stock price in the medium term gradually decreases. During economic downturns, green bonds and clean energy can boost up stock prices. The impacts of the fluctuation of a clean energy market on the green bonds and stock prices differ in lag periods pre- and during COVID-19. In these three periods, the global stock market significantly and negatively impacts clean energy and the green bonds.

The contributions of our analysis mainly lie in three aspects. First, we propose suggestions for policymakers to accelerate energy transition, strengthen supervision and improve green financial system to ensure the development of green economy. Second, our analysis focuses on the nonlinear and time-varying characteristics between green bonds, clean energy, and stock prices, which can help investors adjust investment decisions according to different situations. Third, our empirical results suggest enterprises can achieve sustainable economic development to resume production relying on green subsidies and green credit policies.

The paper is mainly divided into the following parts. Section 2 displays the literature review. In Sect. 3, we describe the data and method. In Sect. 4, we discuss the results. Section 5 is the conclusion and suggestion. 


\section{Literature review}

In recent decades, the green economy has been widely proposed and attracted much attention, attributed to the destruction of natural resources and the increase in carbon emissions caused by economic development at the expense of the environment and resources (Benedetti et al., 2019). The green economy deals with issues related to unsustainable economic development and environmental deterioration. The green economy, interpreted as improving human well-being and society equity and reducing environmental and ecological issues significantly (United Nations Environment Programme, UNEP, 2010), aims at low-carbon development, which can achieve low energy consumption, high efficiency, and low carbon emission, meanwhile, changing human life for the better. The green economy has widely been applied around the world (Loiseau et al., 2016; Wu et al., 2020). Transition to green economy requires the use of appropriate financial tools to invest in a large number of long-term financial resources. Green bonds have proven to be the best financial tools to finance green development (Monasterolo \& Raberto, 2018). Green bonds refer to bonds focusing on environmental protection projects, such as bonds related to clean energy, energy efficiency and low-carbon transportation (Campiglio, 2016). Over the past few year, green bond market has developed rapidly. As of 2019, green bonds have been issued in 62 countries (Initiative, 2019). Between 2016 and 2019, China's issuance of green bonds has exceeded RMB 1.1 trillion, which makes it one of the world's largest green bond market (Huang \& Yue, 2020). As an effective means to finance and refinance the green development, green bond financing contributes to the transition to clean energy. Since clean energy has advantages of reducing carbon emissions and improving energy efficiency, it plays a critical role in sustainable development (Dincer $\&$ Acar, 2015). Therefore, clean energy investment and consumption have attracted more and more attention (Strantzali \& Aravossis, 2016; Wu, Wang, et al., 2020). Many countries around the world are introducing various clean energy policies to promote the development of clean energy, such as solar and wind energy (Liu \& Zeng, 2017; Zhang et al., 2020), and are adopting measures to accelerate the transition to clean energy ( $\mathrm{Li}$ et al., 2021). It is estimated that global solar, wind, and hydropower will account for $62 \%$ of total energy production, and fossil fuel will decline by $31 \%$ by 2050 (BloombergNEF, 2020). The changes of energy structure make a significant contribution to the development of green bond market. A growing literature focuses on the relationship between green bond and clean energy. When Liu, Wei, et al. (2021) estimate the dependence between green bonds and clean energy, they find there exists a positive connectedness between clean energy and green bonds. Nguyen et al. (2021) employ the Rolling Window Wavelet Correlation and find green bonds display a high correlation with clean energy. There also exist contrary conclusions. Hammoudeh et al. (2021) investigate the relationship between green bonds and clean energy index, showing that causality from clean energy index to green bonds is limited. Ferrer et al. (2021) consider the time-varying connectedness between global green bond market and clean energy market, the results indicate there exists a quite limited connectedness. Some literature also pays attention to the connectedness between green bond and clean energy during normal and extreme market conditions. Pham (2021) employ a cross-quantilogram framework to examine the dependence between green bonds and clean energy, they find the connectedness between green bonds and clean energy has been strengthened during extreme market conditions. It is worth noting that the dependence between green bonds and clean energy is time-varying. Therefore, we examine the connectedness between the green bonds and clean energy in the short, medium, and long term by a time-varying model. 
As the "economic barometer" (Borjigin et al., 2018), clean energy can also influence stock price. External factors, such as energy policy, extreme weather, and supply and demand may cause the volatility of the clean energy market, which will influence the enterprises relying on clean energy. Thus, the stock market reacts to the changes in the real economy. Lin et al. (2019) apply MS-VAR-cDCC-FIAPARCH and conclude that a Granger causality from natural gas prices to the Chinese stock market exists. Uddin et al. (2019) employ a crossquantilogram approach to investigate the dependence between clean energy stock returns and aggregate stock returns. The results show that clean energy returns have positive effects on the aggregate stock index, and the effects are asymmetric across quantiles. Using the wavelet correlations method, Urom et al. (2021) demonstrate that the U.S. and European clean energy markets can positively influence global stock returns in the short term, however, the impacts are weak. The results also show that the positive effects of the Asian clean energy market are close to zero in the short term.

The stock market plays a crucial part in allocating capital in green projects (Paramati et al., 2016), which affects the clean energy market. With the increasing awareness of global lowcarbon development, clean energy has become an important substitute for traditional fossil energy (Yildirim, 2014). Previously there have been many studies focusing on the nexus between clean energy and stock price (Al Mamun et al., 2018; Elsayed et al., 2020; Geng et al., 2021; Kuang, 2021; Lin et al., 2019; Paramati et al., 2016; Razmi et al., 2020; Urom et al., 2021). Two strands mainly separate the results. One strand is the positive impacts of stock prices on clean energy. Paramati et al. (2016) employ the Autoregressive Distributed Lags approach to investigate the impact of stock market development on clean energy, and they find that the development of the stock market exerts a positive impact on clean energy use. Employing the sample data of 25 Organization for Economic Co-operation and Development (OECD) countries, Al Mamun et al. (2018) argue that the development of the stock market can significantly and positively influence cleaner energy production. Elsayed et al. (2020) use the Diebold and Yilmaz time-domain connectedness measures to examine volatility spillover between stock price index and clean energy price index and find the high volatility of the MSCI World Stock Price Index contributes to the fluctuation of the WilderHill Clean Energy Price Index. Using an autoregressive distributive lag (ARDL) model, Razmi et al. (2020) discover the positive relationship between stock price and the clean energy market. Another group of scholars concludes the negative impacts of stock prices on clean energy. Urom et al. (2021) study the dependence between the global stock market and the clean energy market. They conclude that an increase in global stock market volatility can result in a decrease in clean energy stock prices in the U.S., Europe, and Asia.

Stock prices can not only influence clean energy, but they also have connectedness with green bonds. Nguyen et al. (2021) examine the time-frequency co-movement between green bonds and stocks, the results reveal the negative correlations between green bonds and stocks. Wang et al. (2020) conclude that stock returns response positively to green bonds. Gao et al. (2021) employ a multidimensional DCC-GJRGARCH model to explore the spillover between green bond and stock market. They find industrial stock markets generate unidirectional risk spillovers to green bond markets. However, Reboredo (2018) documents that green bonds weakly co-move with stock markets. Reboredo and Ugolini (2020) also conclude that green bonds weakly connect with stock markets.

Despite the results drawn by the above literature, note that most of it has ignored the nonlinear relationship between the green bonds, clean energy, and global stock market (Paramati et al., 2016; Razmi et al., 2020). Traditional methods cannot capture the dynamic change of the relationship. If the non-linear relationship between variables is ignored, some important characteristics among variables may be ignored (Piotr \& Witold, 2018). Moreover, an 
important point that has been ignored in certain literature is the lack of considering different economic conditions. Especially in economic crisis, statistical bias will occur (Kumah, 2007). The standard VAR model with constant parameters allows drawing impulse responses only for variables under the assumption that parameters do not change at different horizons and points of the impulse responses. However, due to time-varying state in variables, it may result in inaccurate results. Compared to the traditional VAR model, the coefficient of the TVP-VAR model and covariance matrix are time-varying (Esmaeili \& Rafei, 2021). Based on maintaining the advantages of the VAR model, the TVP-VAR model liberates the limit of model coefficient and disturbance term, providing a reliable research framework for describing the nonlinear dynamic characteristics of variables. Furthermore, multivariate random fluctuations can reflect the possible heteroscedasticity and the TVP-VAR model can capture the different economic conditions (Esmaeili \& Rafei, 2021). The introduction of time-varying parameters improves the fitting accuracy of the model and increases the explanatory power of the model (Chatziantoniou et al., 2021; Gong et al., 2021; Gu et al., 2021). Chatziantoniou et al. (2021) provide evidence that TVP-VAR model is more reliable during severe episodes of declining economic activity. Gong et al. (2021) confirm the advantages of TVP-VAR in analyzing dynamic correlation between variables. Therefore, we introduce the TVP-VAR model (Primiceri, 2005) to solve the time-varying relationship. Professionals in the economic field have widely used the TVP-VAR model (Adekoya \& Oliyide, 2021; Baumeister \& Peersman, 2013; He, 2020; Nakajima, 2011; Urom et al., 2020; Zhou et al., 2020). Baumeister and Peersman (2013) study the impact of oil supply on the American economy through TVP-VAR. He (2020) employs the TVP-VAR model to analyze the time-varying relationship between oil price fluctuations and investor sentiment. In addition, the TVP-VAR model is also used to study the dynamic dependence of the Bitcoin market, securities market, gold market, and crude oil market (Urom et al., 2020).

Extensive studies have focused on green bonds, clean energy, and the stock market. In this paper, given the shock of the COVID-19 pandemic, we argue that the TVP-VAR model serves as a suitable tool to consider the nonlinearities. Therefore, we employ such a model to shed light on the dynamic connectedness between the green bonds, clean energy, and stock prices.

\section{Data and methodology}

\subsection{Data}

\subsubsection{Sample selection and data source}

This paper investigates the nexus between green bonds, clean energy and stock price. S\&P Green Bond Index (denoted by SPGB) is selected to represent the performance of green bands market (Nguyen et al., 2021). To reflect the clean energy (denoted by SPCE), we employ the S\&P Global Clean Energy Index (Dawar et al., 2021; Liu, Wei, et al., 2021; Nguyen et al., 2021). The MSCI ACWI Index is designed to represent the performance of large and mid-cap stocks in 23 developed markets and 26 emerging markets. The index has covered more than 3000 constituent stocks in 11 industries, accounting for approximately $85 \%$ of the free-floating adjusted market value of each market (Reboredo, 2018). Therefore, we use the MSCI ACWI Index (denoted by MSCI) to represent the stock price. The S\&P Green Bond Index is available at S\&P Global (https:/www.spglobal.com/spdji/en/indices/esg/sp-green- 

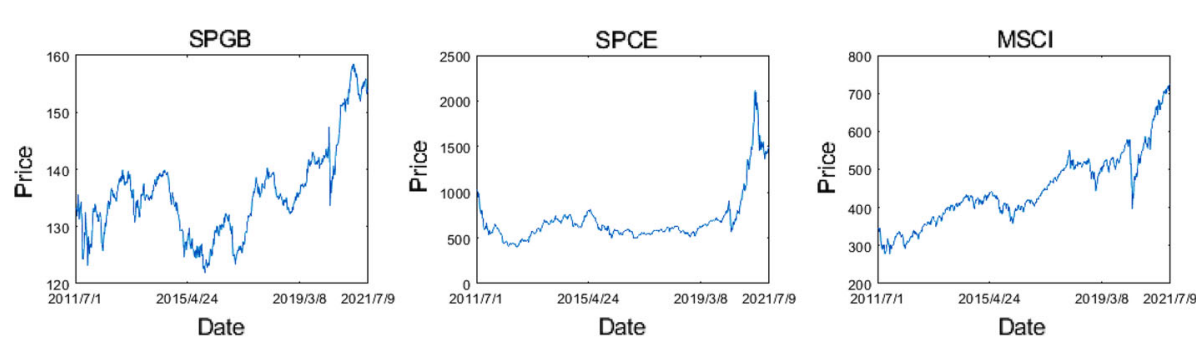

Fig. 2 Dynamics of S\&P green bond index, S\&P global clean energy index and MSCI world index price

bond-index/\#overview). The S\&P Global Clean Energy Index and MSCI ACWI Index are from the WIND database. The weekly data are from July 1, 2011 to July 9, 2021.

Figure 2 displays the weekly data of the S\&P Green Bond Index, S\&P Global Clean Energy Index and MSCI ACWI Index. The S\&P Green Bond Index showed a violent fluctuation, especially in 2015, the index fell sharply. After that, the index showed an upward trend. In March 2020, the S\&P Green Bond Index dropped sharply to about 130 USD due to the shock of COVID-19 pandemic, and then the index increased sharply. The S\&P Global Clean Energy took on an upward trend pre-COVID, but the index has changed smaller since 2021. During the COVID-19 pandemic, the stock market triggered circuit breakers, the S\&P Global Clean Energy Index dropped sharply. And then, the index rose sharply. Till January 2021, the index reached maximum. Before COVID-19 pandemic, the fluctuation of the MSCI ACWI Index showed downward trend initially, and then the index increased gradually. However, during the COVID-19 pandemic, the circuit breakers tripped for four times, and the MSCI ACWI Index suffered a sharp fall. And then, the MSCI ACWI Index increased dramatically, which is closed to 700 USD eventually.

\subsubsection{Descriptive statistics}

The descriptive statistics of the S\&P Green Bond Index, the S\&P Global Clean Energy Index and the MSCI ACWI Index is displayed in Table 1. The skewness of SPGB, SPCE and MSCI is greater than 0, which means the data is biased to the right. The kurtosis of SPGB, SPCE, and MSCI is greater than 3, thus the data exhibits the characteristics of sharp peaks and thick tails. The results of Jarque-Bera test reject the null hypothesis of non-normal distribution.

Table 1 Descriptive statistical results of variables

\begin{tabular}{lrrr}
\hline Statistics & \multicolumn{1}{c}{ SPGB } & \multicolumn{1}{c}{ SPCE } & \multicolumn{1}{c}{ MSCI } \\
\hline Mean & 135.710 & 696.149 & 447.538 \\
Median & 135.130 & 613.825 & 427.130 \\
Maximum & 158.510 & 2113.520 & 724.660 \\
Minimum & 121.880 & 399.340 & 277.840 \\
Std. Dev & 7.921 & 281.251 & 95.598 \\
Skewness & 0.914 & 2.661 & 0.680 \\
Kurtosis & 3.736 & 10.330 & 3.320 \\
Jarque-Bera & 84.415 & 1784.847 & 42.507 \\
Probability & 0.000 & 0.000 & 0.000 \\
\hline
\end{tabular}




\subsubsection{Stationary test}

The unit root test results of Augmented Dickey-Fuller (ADF) (Fuller, 1979) and PhillipsPerron (PP) (Phillips \& Perron, 1988) are presented in Table 2. The results show that the time series of SPGB, SPCE and MSCI are non-stationary, while they are stationary after the process of first-order logarithmic difference. Therefore, we employ the first-order logarithmic difference of the data to the TVP-VAR model.

\subsubsection{Nonlinearity test}

In this paper, the Brock-Decher-Scheikman (BDS) test was performed on the nexus between variables. The BDS test results showed in Table 3 demonstrate that SPGB, SPCE, and MSCI all showed a nonlinear relationship. The reasons can be explained as follows. There exists certain cycle in the operation of economy, which will affect investor sentiment and enterprises' performance. Generally speaking, investors' income increases in a prosperous or recovering period, leading to more positive sentiment among investors and more enthusiasm for highrisk products, which will cause market participants to invest funds in stocks. On the contrary, investors' income decreases in a period of recession or depression, causing investors to feel pessimistic. At this time, investors are keen on low-risk products, and they are more likely to invest funds in lower-risk but lower-yield products, thus causing funds to gradually enter green bonds. As for enterprises, green bonds may have different impacts on corporate performance in different economic periods, the issuance of green bonds may help environmentally friendly enterprises resume production during the recession, resulting in better performance in stock market. More and more countries focus on the development of green economy, which leads to the improvement of green bond system. Thus, the performance of green bonds may show significant difference in different period, which will further cause the difference of the investment and consumption of clean energy supported by green bonds. Similarly, there also exists obviously difference of the relationship between clean energy and stock price in different stage. Therefore, we use the TVP-VAR model to solve the nonlinear relationship between green bonds, clean energy and stock price.

\subsection{Methodology}

The TVP-VAR model is derived from the structural VAR (SVAR) model, which is given by,

$$
A y_{t}=F_{1} y_{t-1}+\ldots+F_{s} y_{t-s}+\mu_{t}, \quad t=s+1, \cdots, n
$$

where $y_{t}$ is the $n \times 1$ vector of response, $A$ and $F_{1} \cdots F_{s}$ are $n \times n$ time-varying matrices of coefficients. The disturbance $\mu_{t}$ is a $n \times 1$ structural shock, which is assumed to follow the normal distribution, denoted $\mu_{t} \sim N(0,66)$, where $\Sigma$ is the diagonal matrix,

$$
\Sigma=\left(\begin{array}{cccc}
\sigma_{1} & 0 & \cdots & 0 \\
0 & \sigma_{2} & \ddots & 0 \\
\vdots & \ddots & \ddots & 0 \\
0 & \cdots & 0 & \sigma_{n}
\end{array}\right)
$$




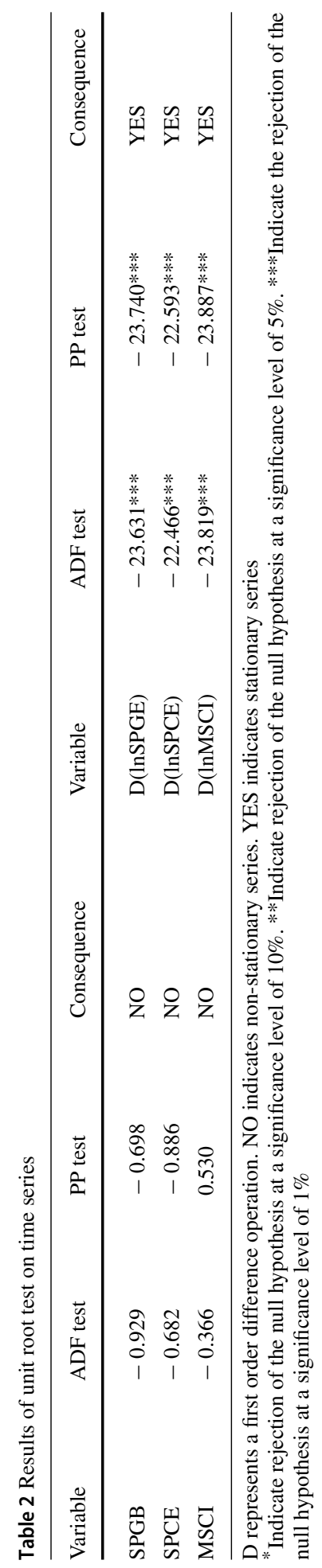




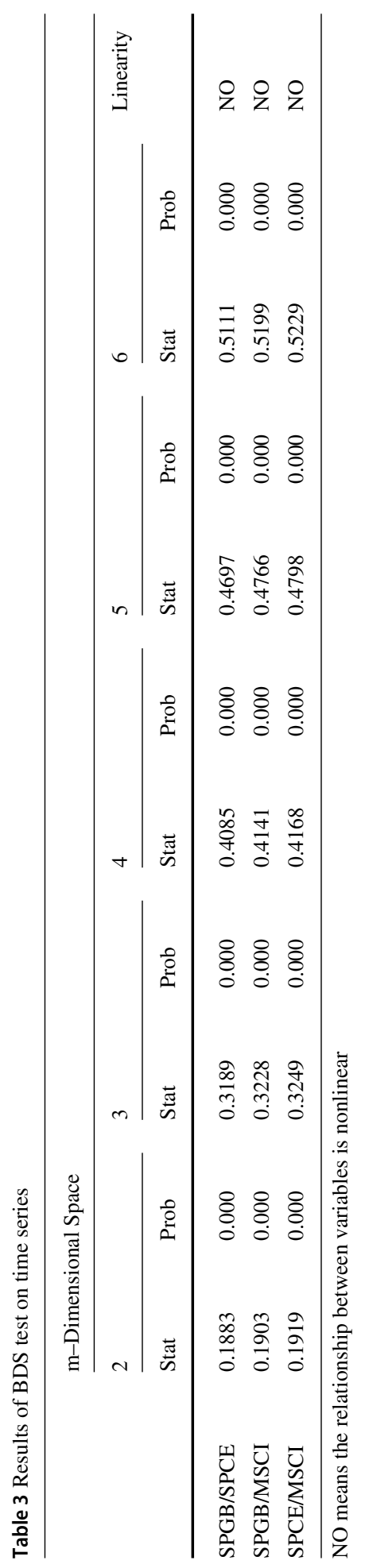


Assuming $A$ is lower-triangular whose main diagonal is 1 , Eq. (1) can be rewritten into a recursive form,

$$
y_{t}=B_{1} y_{t-1}+\cdots+B_{s} y_{t-s}+A^{-1} \Sigma \varepsilon_{t}, \varepsilon_{t} \sim N\left(0, I_{n}\right)
$$

where $B_{i}=A^{-1} F_{i}, i=1, \cdots, s$. Stacking the elements in the rows of the $B_{i}$ 's to form $\beta$ $\left(k^{2} s \times 1\right)$. Equation (3) can be written as,

$$
\begin{gathered}
y_{t}=X_{t} \beta+A^{-1} \Sigma \varepsilon_{t} \\
X_{t}=I_{n} \otimes\left(y_{t-1}^{\prime}, \cdots, y_{t-s}^{\prime}\right)
\end{gathered}
$$

where $\otimes$ represents the Kronecker product. Therefore, the TVP-VAR can be written as,

$$
y_{t}=X_{t} \beta_{t}+A_{t}^{-1} \Sigma_{t} \varepsilon_{t}, t=s+1, \cdots, k
$$

The coefficient $\beta_{t}$, the simultaneous parameter matrix $A_{t}$ and the covariance of random fluctuations $\Sigma_{t}$ are time-varying.

According to the method of Primiceri (2005) and Nakajima (2011) that processes the time-varying parameters, the non-zero elements and elements with a value of 1 in the lower triangular matrix $A_{t}$ are stacked into a column of vectors, namely $\alpha_{t}=$ $\left(\alpha_{21}, \alpha_{31}, \alpha_{41}, \cdots, \alpha_{n, n-1}\right)^{\prime} \cdot h_{t}=\left(h_{1 t}, \cdots, h_{n t}\right)^{\prime}$, where $h_{j t}=\log \sigma_{j t}^{2}, j=1, \cdots, n ; t=$ $s+1, \cdots, k$. According to Nakajima (2011), the parameters to be estimated follow the random walk process: $\beta_{t}=\beta_{t-1}+\mu_{\beta t}, a_{t}=a_{t-1}+\mu_{a t}, h_{t}=h_{t-1}+\mu_{h t}, t=s+1, \cdots, k$, where $\beta_{s+1} \sim N\left(\mu_{\beta_{0}}, \Sigma_{\beta_{0}}\right), \alpha_{s+1} \sim N\left(\mu_{\alpha_{0}}, \Sigma_{\alpha_{0}}\right), h_{s+1} \sim N\left(\mu_{h_{0}}, \Sigma_{h_{0}}\right)$. The model's variance-covariance matrix is a block diagonal,

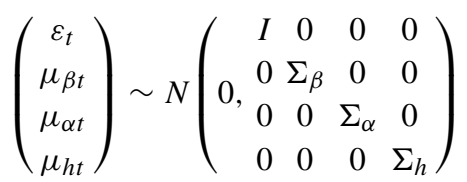

where I is a n dimensional identity matrix, $\Sigma_{\beta}, \Sigma_{\alpha}$ and $\Sigma_{h}$ are positive definite matrices.

According to Nakajima (2011), the MCMC method is employed for estimation to overcome the limitation that the likelihood function is difficult to solve due to the non-linear random fluctuations.

\section{Results}

Since the time-varying coefficient model has stronger explanatory power than the nonlinear model (Granger, 2008), we introduce the TVP-VAR model to investigate the dynamic nexus between green bonds, clean energy, and stock price. According to He (2020), we choose the optimal lag order of the TVP-VAR model to be 4 based on AIC.

\subsection{Estimated results under the MCMC simulation method}

The Markov Chain Monte Carlo algorithm (MCMC) in the context of Bayesian inference simulates 50,000 times, and the result of the first 5000 iterations is a burn-in sample. The MCMC estimation results of parameters are displayed in Table 4. From the convergence perspective, the Geweke'CD statistics of all variables are less than the critical value (1.96) 
Table 4 MCMC estimation results of parameters

\begin{tabular}{llllllr}
\hline Parameter & Mean & SD & $95 \% \mathrm{~L}$ & $95 \% \mathrm{U}$ & Geweke'CD & \multicolumn{1}{c}{ Inef. } \\
\hline$\left(\Sigma_{\beta}\right)_{1}$ & 0.0023 & 0.0003 & 0.0018 & 0.0029 & 0.736 & 27.11 \\
$\left(\Sigma_{\beta}\right)_{2}$ & 0.0023 & 0.0003 & 0.0018 & 0.0028 & 0.180 & 27.03 \\
$\left(\Sigma_{\alpha}\right)_{1}$ & 0.0057 & 0.0017 & 0.0034 & 0.0098 & 0.855 & 143.81 \\
$\left(\Sigma_{\alpha}\right)_{2}$ & 0.0178 & 0.0114 & 0.0042 & 0.0434 & 0.003 & 286.39 \\
$\left(\Sigma_{h}\right)_{1}$ & 0.2261 & 0.0376 & 0.1588 & 0.3070 & 0.889 & 70.76 \\
$\left(\Sigma_{h}\right)_{2}$ & 0.2311 & 0.0364 & 0.1672 & 0.3089 & 0.153 & 64.21 \\
\hline
\end{tabular}

at the 5\% significance level. Therefore, the null hypothesis of convergence to the posterior distribution cannot be rejected, which indicates effective results. As for inefficiency factors, the inefficiency factors of $\left(\Sigma_{\alpha}\right)_{1}$ and $\left(\Sigma_{\alpha}\right)_{2}$ are 143.81 and 286.39 , respectively, which are much less than the fifty thousand sampling times. The remaining inefficiency factors are all less than one hundred, indicating that the sample obtained by MCMC sampling fifty thousand times is convergent. Therefore, the simulated sampling results are ideal, and the samples obtained by MCMC sampling are sufficient for the TVP-VAR model.

Figure 3 shows that the sample autocorrelations show a steady downward trend, and sample paths are stable, indicating that our sampling method efficiently produces the samples with low autocorrelation. The results show that the simulation process is effective and robust, and the simulation results converge to the posterior distribution.

Figure 4 displays the posterior distribution of the random fluctuations of three variables. In 2011, the S\&P Green Bond Index fluctuated dramatically. And then, the fluctuation reached its minimum and kept in a low level. The volatility peaked in 2020 , which may be attributed to the negative impact of COVID-19. After that, the fluctuation declined and remained low. Due to the influence of COVID-19, the S\&P Global Clean Energy Index fluctuated violently.
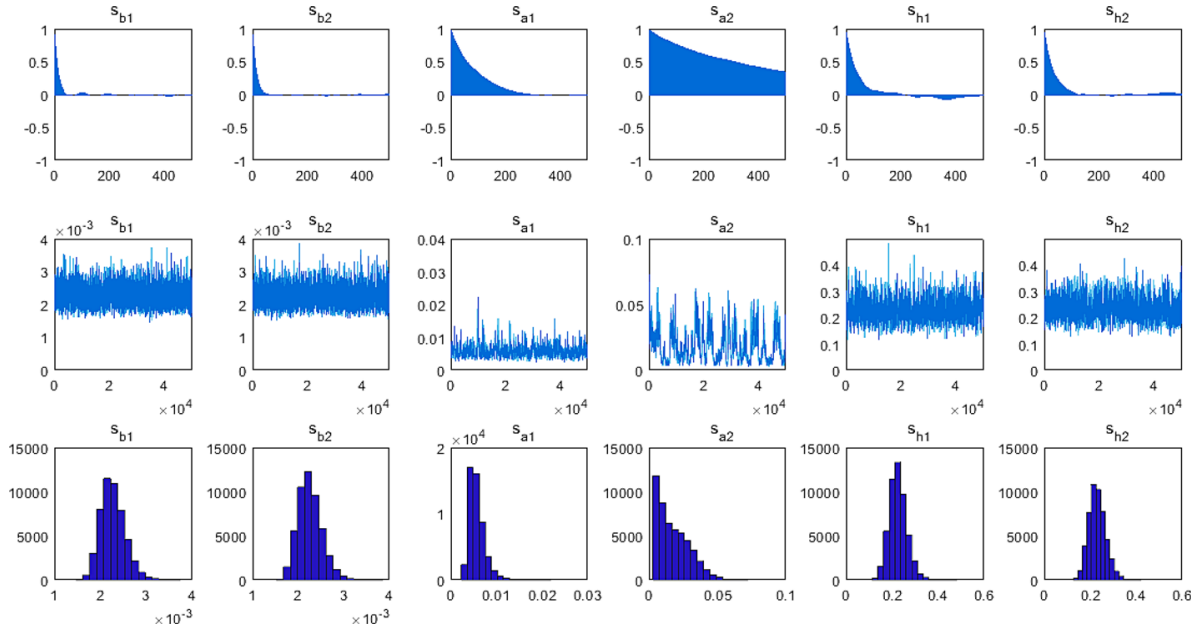

Fig. 3 The autocorrelation coefficient, sample path and posterior distribution 

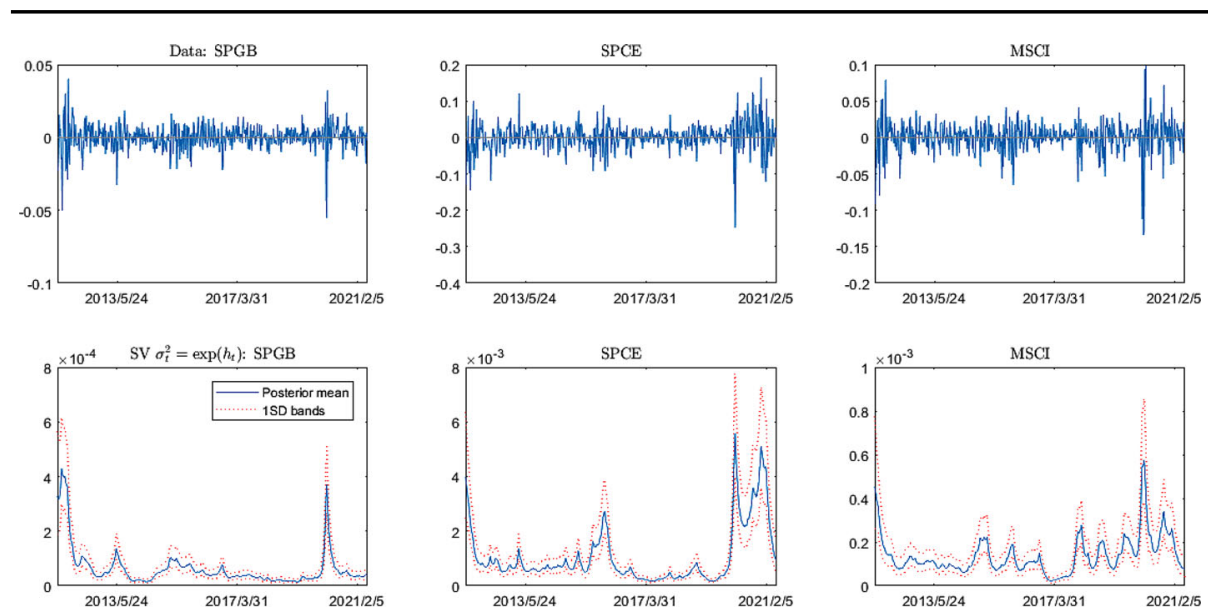

Fig. 4 Posterior estimation for stochastic volatility of the structural shock
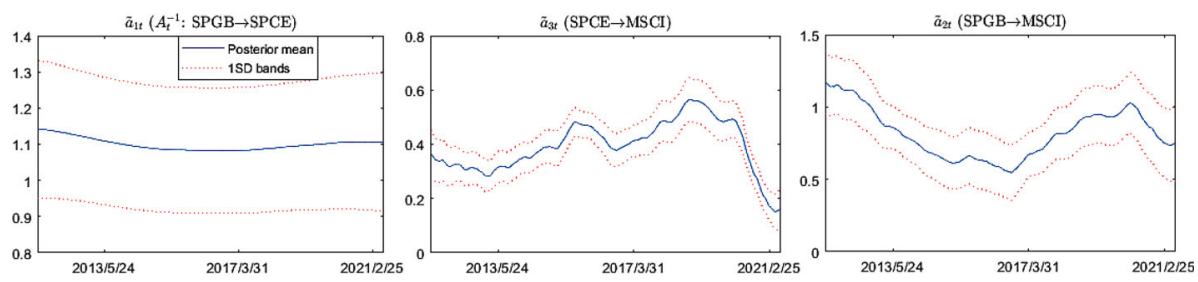

Fig. 5 Simultaneous relation for SPGB, SPCE and MSCI

Subsequently, the fluctuation declined and reached its lowest level in May 2020. The COVID19 pandemic also caused the MSCI ACWI index to fluctuate sharply in March 2020, and then its volatility has declined, but the volatility is more severe than before March 2020 .

We employed the TVP-VAR model to explain the time-varying relationship between variables. According to Eq. (6), The lower triangular matrix $A_{t}$ explains the simultaneous relation between variables. The posterior estimation $\tilde{\alpha}_{i t}$ of the free elements in $A_{t}^{-1}$ is displayed in Fig. 5. Firstly, the results show that the simultaneous relation between the green bonds and clean energy is positive, and the positive relationship keeps in a high level, attributed to the fact that green bonds can raise funds for green projects, especially for clean energy, which can accelerate the transformation of energy structure. Since countries around the world are paying more attention to green development and the green financial system is gradually improving, the green bonds is playing a vital important role in clean energy. Second, the green bonds have a positive impact on stock price. The positive impact of the green bonds on the stock price decreased before 2017. This could be because that green bond markets have attracted more funds from stock price in the initial stage, which leads to decreasing trend of the positive impact. In 2015, the stock market suffered from a crisis. And the global economic downturn has led to a decline in the scale of green bond issuance, which further exacerbates the downward trend of the positive impacts. After 2017, there exists an increased trend of the positive effects of green bonds on stock price. This can be attributed to the fact that China has taken active measures to participate in green bond market since 2017, and the issuance of green bonds has increased significantly, which contributes to the production of 
environmentally friendly enterprises. During the COVID-19 pandemic, the positive effects of the green bonds on stock price decreased. This may be because that enterprises have suffered huge losses due to the COVID-19 pandemic. Third, clean energy positively influenced stock prices, consistent with Razmi et al. (2020). The positive effects can be explained by the fact that governments around the world take active measure to develop green economy to resume production. Before the COVID-19 pandemic, the positive effects were stable and remained in high level. However, the positive impacts weakened after the outbreak of COVID-19 pandemic, attributed to the fact that enterprises reduced productions, resulting in the less demand for energy. Therefore, the effects of clean energy on stock prices decreased. Therefore, the acceleration of the development of clean energy on stock price weakens.

\subsection{Time-varying impulse response of different time horizons}

Figures 6, 7 and 8 presents the impulse responses for a 2-week horizon, 4-week horizon, and a 6-week horizon to represent the short term, medium term, and long term, respectively (Kassouri et al., 2021). Figure 6 shows that, in the short term, the green bonds can positively impact clean energy, and the positive impacts have increased before 2017, which can be attributed to the development of green bond markets. From 2017 to 2019, the positive impacts have decreased. After the outbreak of COVID-19 pandemic, the positive impacts also increase.
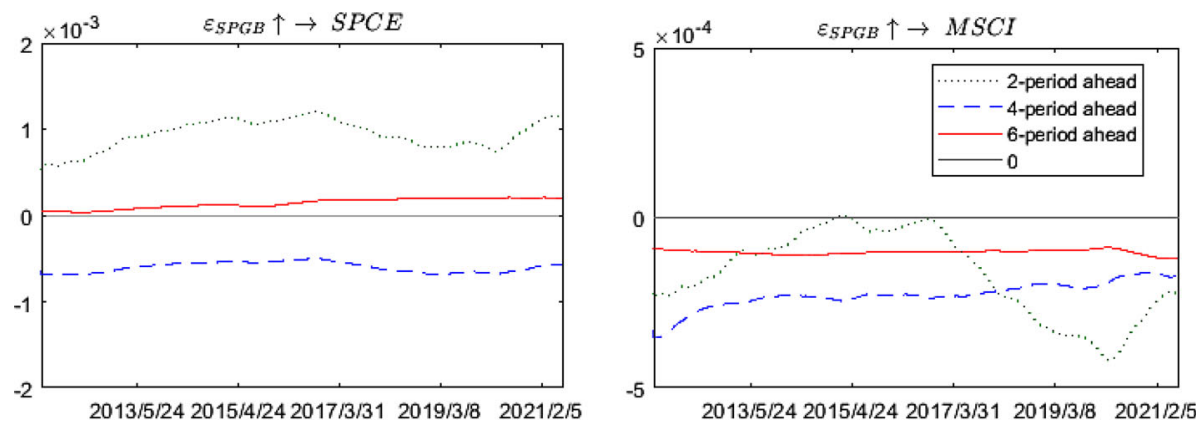

Fig. 6 Time-varying response of SPCE and MSCI to SPGB
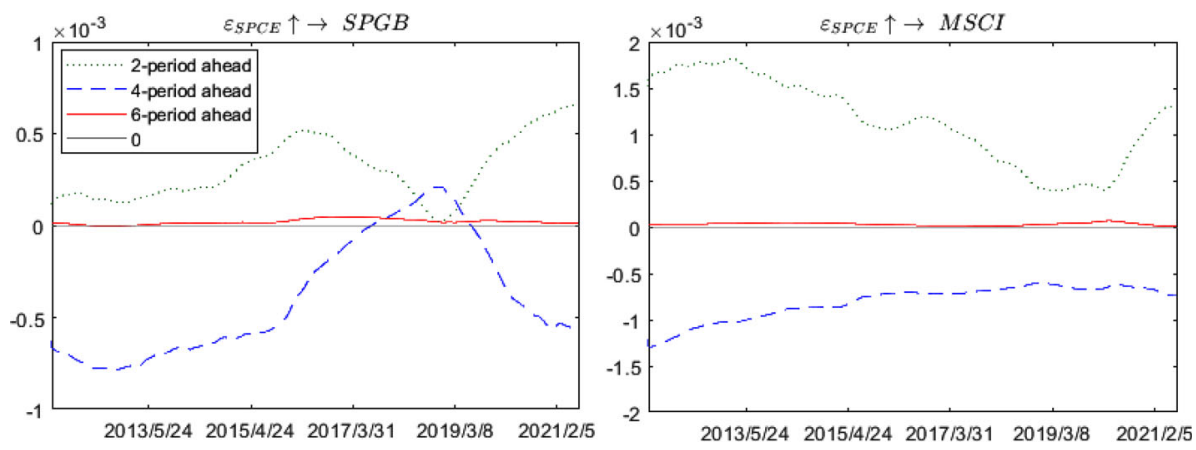

Fig. 7 Time-varying response of SPGB and MSCI to SPCE 

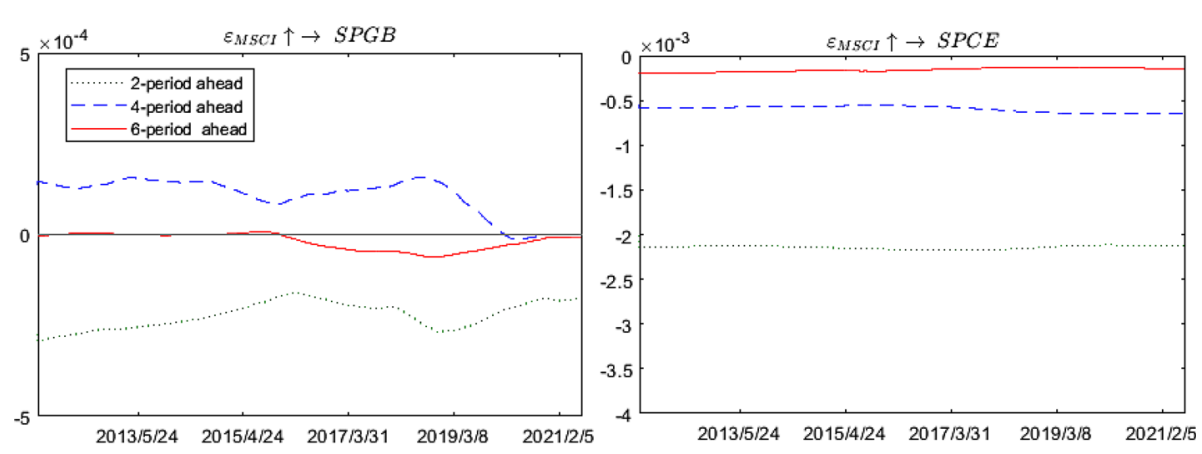

Fig. 8 Time-varying response of SPGB and SPCE to MSCI

This may be because that governments around the world pay more attention to green development to recover the economy undermined by the COVID-19 pandemic, which leads to the increased demand for clean energy. Therefore, the development of green bond market due to the incentive policies can encourage the enterprises to use more clean energy. In the medium term, the impact of green bonds on clean energy is negative. The negative influences of green bonds on clean energy are almost unchanged. The clean energy sector is the main investment area for green bonds, however, funds raised from green bonds may be used for other green projects, such as sewage treatment. In the medium term, governments and enterprises may pay more attention to other green projects to properly allocate green resources, which results in the less financial supports for clean energy market. Moreover, enterprises may pay more attention to technological innovation to achieve green production. Thus, enterprises may increase investment in technological innovation, and reduce the investment in clean energy. In the medium term, the green financing of enterprises may cut the investment in the high-risk clean energy market (He et al., 2019), thus proving that green bonds can negatively impact clean energy. Therefore, the funds flowing to clean energy market may be reduced, resulting in the negative effects on clean energy. In the long term, the green bonds have a positive impact on clean energy initiative, and the positive impact increases. However, the positive impacts in the long term are weaker than the positive impacts in the short term.

In the short, medium and long term, the green bonds negatively impact stock prices, which is consistent with Nguyen et al. (2021). The reason for this could be that the development of green bond market appeals more investors from stock market. However, the negative impacts in the long term are weaker than that in the medium term. In the short term, the negative impacts have decreased before 2015. Between 2015 and 2017, the negative impacts are closed to zero. This may be due to the fact that the 2015 stock market crisis disrupted the normal operation of the stock market. During the COVID-19 pandemic, there is a decreasing trend in the relationship between green bonds and stock price. This may be due to the fact that enterprises may pay more attention to green financial instruments to raise funds to resume production. In the medium term, the negative impacts of green bonds on stock price are greater than that in the short term before 2017. The negative effects have decreased gradually, which may be because the global green bond markets continue to improve. Therefore, the inhibiting effect of the green bonds on stock price will weaken.

The impact of clean energy on the green bonds and stock prices is shown in Fig. 7. Clean energy has positive effects on green bonds. There is a sharp decrease in the positive effects of clean energy on green bonds between 2017 and 2019. During this period, the share of energy in the allocation of green bonds dropped, resulting in weaker positive effects (Initiative, 2019). 
During the COVID-19 pandemic, clean energy exerts an increasingly positive impacts on green bonds. The reason for this could be that the green bonds market is mainly influenced by enterprises' operational status and supply-demand relationship. To response to the green development and recover production, enterprises will raise more funds to support the green development. In the medium term, the development of clean energy exerts a negative impact on the green bonds before 2018, and the negative impact decreases over time. Between 2018 and 2019, clean energy can positively influence green bonds, which can be attributed to the development of clean energy technology, resulting in more demand for green bonds. The dynamic relationship between clean energy and green bonds significantly increases during the COVID-19 pandemic. In the long term, clean energy has positive effects on green bonds, however, the effects are slight.

Figure 7 shows that clean energy exerts positive impacts on stock prices in short term. There is a decreasing trend of the negative impacts before 2019. During the COVID-19, the positive effects increase over time. The results indicate that clean energy can contribute to resuming the production and recovering the economy in the extreme market conditions. In the medium term, clean energy can negatively impact stock prices. This may be attributed to the high cost of clean energy. To achieve their targets of emission reduction, companies will invest large amounts of capital in clean energy, which will hinder the development of other fields, adversely affecting production. With development of clean energy market and the maturity of clean technology, the negative impacts decline over time. In the long term, the fluctuation of clean energy will promote the stock price, possibly due to the improvement of the green financial system and the rapid development of the clean energy market. More and more enterprises attach importance to clean energy, which promotes investment and consumption in clean energy. Furthermore, in the long term, using clean energy reduces the cost of pollution control in the production of enterprises, which produces better environmental benefits. This is conducive to promoting enterprises' production.

As shown in Fig. 8, the increase of the stock prices negatively influences the green bonds in the short term. This may be because the increase in stock prices encourages enterprises to expand production. However, this may cause enterprises to neglect environmental governance, which hinders the development of a green bonds market. In the medium term, stock prices can exert a positive impact on the green bonds. The improvement of stock prices promotes enterprises to enlarge production, and pay more attention to green production. Therefore, enterprises will raise more funds through green bonds, which promote the development of green bond market. However, the positive impact has been closed to zero since 2020. From the perspective of investors, the stock market has suffered a huge loss, which may cause the investors to lose confidence in stock and bond market, and investors will reduce the investment in two markets. As for enterprises, the global economy has suffered a severe setback due to the COVID-19 pandemic, a large number of enterprises reduce production to reduce production to reduce losses. Moreover, enterprises reduce their investment in low-carbon development, resulting in decreasing influence.

In the short, medium and long term, stock prices negatively impact clean energy. The negative impacts at three period horizons are almost unchanged. Sari et al. (2008) also found the improvement of industrial output can hinder the development of the clean energy market. This may be because the improvement of stock prices contributes to the expansion of production, which results in more consumption of non-clean energy. The negative impacts are greater in the short term than that in the other two horizons. 


\subsection{Impulse analysis of different points}

We further analyze the impulse response at different points, shown in Figs. 9, 10 and 11. On December 31, 2019, Wuhan had outbreak from unknown virus. On January 12, 2020, World Health Organization (WHO) announced the fast-spreading virus to be "2019-nCoV", which was further proclaimed to be COVID-19 on February 11, 2020. Countries around the
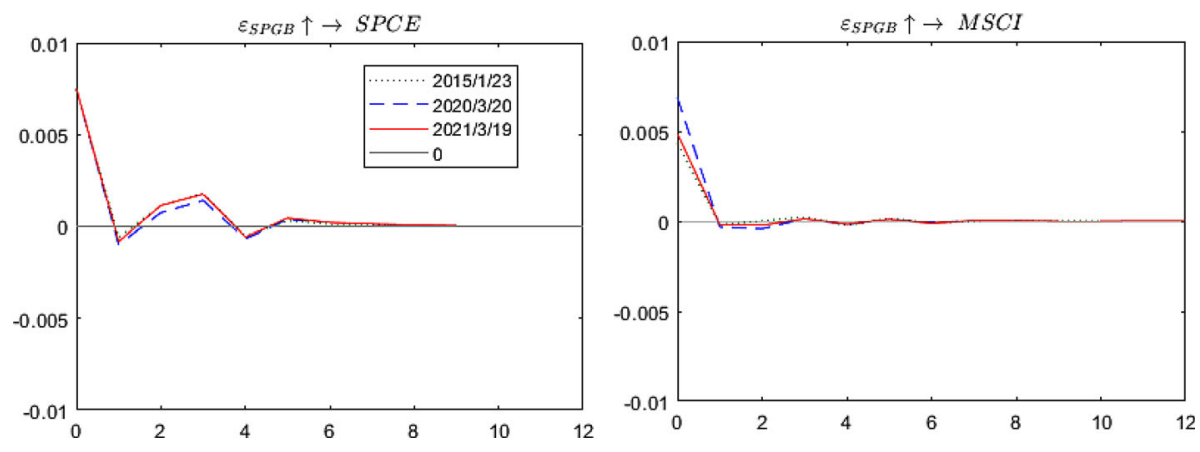

Fig. 9 Responses of SPCE and MSCI to SPGB in different points
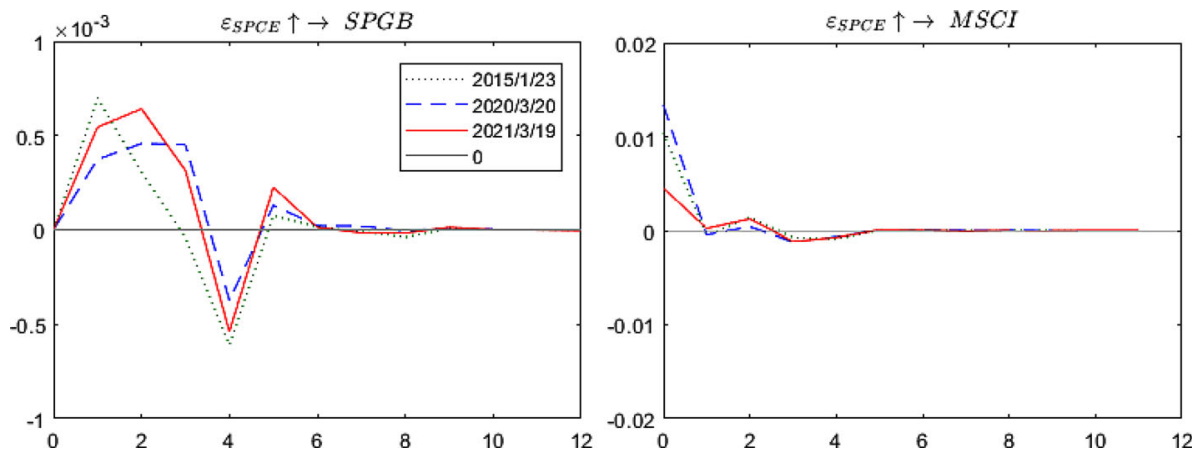

Fig. 10 Responses of SPGB and MSCI to SPCE in different points
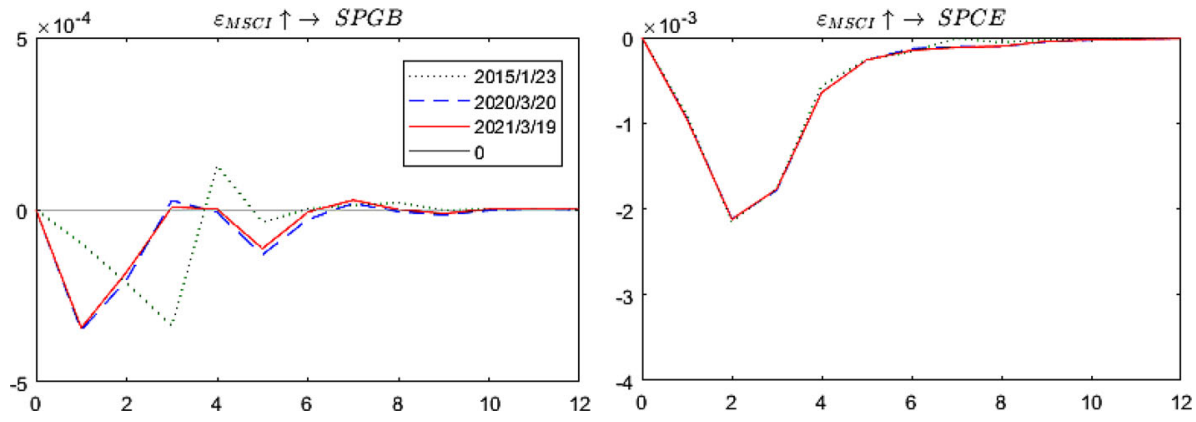

Fig. 11 Responses of SPGB and SPCE to MSCI in different points 
world have taken active measures to fight against the COVID-19, and they have achieved remarkable results. On December 3, 2020, the UN General Assembly held a special session on the COVID-19 pandemic response to discuss the access to vaccine and economic recovery. Therefore, we identify the period prior to December, 2019 as the period before COVID-19 pandemic, the period from December, 2019 to December 3, 2020 as the period of economic downturn during the COVID-19 pandemic. And the period after December 3, 2020 is identified the period of economic recovery during the COVID-19 pandemic. We choose three different points: $2015 / 01 / 23,2020 / 03 / 20$, and 2021/03/29. Since the outbreak of the epidemic, the global economy has been devastated. On March 11, 2020, WHO declared the outbreak of pandemic, and many countries started lockdown to combat with the pandemic in late March (Nundy et al., 2021). Due to the impacts of the COVID-19 pandemic, there exists 18 jumps in the US stock market from February 24, 2020 to March 24, 2020 (Baker et al., 2020). Therefore, we chose March 20, 2020 to represent the point of economic downturn during the COVID-19 pandemic. We chose January 23, 2015 to represent the point before the COVID-19 pandemic. Despite the ongoing of the COVID-19 pandemic, since 2021, with the acceleration of global vaccination and the successive easing of lockdown by various countries (Nundy et al., 2021), global economy is recovering (United Nations, UN, 2021). Therefore, we use March 19, 2021 to represent the point of economic recovery during the COVID-19.

Figure 9 shows the curve of the impact of the green bonds on clean energy and stock prices pre-, during the COVID-19 pandemic. Figure 9 shows that the impulse response at each point gradually converges over time. In the sixth period, the impact of the green bonds on clean energy and the stock market is close to zero. At different points, the impact of the green bonds on clean energy and the stock market has little difference. The green bonds exert a positive impact on the clean energy market and the stock market at three time points. Therefore, during the economic downturn, the development of a green economy contributes to the rise in stock prices. Comparing the pre-COVID-19 pandemic and the period of economic recovery during the COVID-19, the positive impact of the green bonds on the clean energy market is weaker in the third stage in the period of economic downturn during the COVID-19 pandemic. This may be because the measures of economic recovery after the epidemic effectively promote the transformation of the energy structure (International Renewable Energy Agency, IRENA, 2020). Compared to the period of economic recovery during the COVID-19 pandemic and the period before the COVID-19 pandemic, the green bonds have a greater effect on stock prices in the period of economic downturn during the COVID-19 pandemic period. Thus, the development of green bond market can effectively encourage enterprises to expand production.

Figure 10 shows the impacts of clean energy on the green bonds and stock prices before the COVID-19 pandemic, during the COVID-19 pandemic. The influence of clean energy on the green bonds and stock prices varies greatly. The negative fluctuation of clean energy on the green bonds can be attributed to the high risk of the clean energy market (He et al., 2019), which can adversely affect the green bond market. Before the COVID-19 pandemic, the impact of clean energy on green bonds is stronger than that during the COVID-19 pandemic. This may be due to the recession of the economy during the COVID-19 pandemic. Enterprises will reduce their demand for clean energy to reduce costs, which will weaken the effect of clean energy. The positive impacts of clean energy on the green bonds in the period of economic downturn during the COVID-19 pandemic are weaker than before the COVID-19 pandemic and in the period of economic recovery during the COVID-19 pandemic. And the negative impact is also weaker. This may be because in the period of economic downturn during the COVID-19 pandemic, production activities have been suffered heavy losses, resulting in reduced demand for green bonds. Therefore, the impact will be weakened. 
Figure 10 shows that, in the initial stage, the positive impact of the clean energy market on stock prices in the period of economic recovery is weaker than the positive impact during the period of economic downturn and before COVID-19 pandemic. This may be because there are new risks such as secondary epidemics, de-globalization crises, and debt crises in the economic recovery period, which weaken the positive impact of clean energy on stock prices.

Figure 11 displays that stock prices exert negative influences on green bonds before the COVID-19, during the COVID-19 pandemic. The impact of stock prices on green bonds during the economic downturn and economic recovery period has little difference at varying points, and it reaches its maximum in the first period. Stock prices can also positively influence green bonds, and it reaches its maximum in the fourth period. The negative impacts can be attributed to the fact that the rise of stock prices appeals more investors to take part in stock market instead of green bond market, thus hindering the rise of the green bonds.

In the pre- and during- COVID-19 pandemic, stock prices have little difference in their impacts on clean energy. Stock price can negatively impact clean energy. The results are consistent with Sari et al. (2008) who found that the improvement of industrial production can hinder clean energy usage. The negative impact reaches its maximum in the second period. The reason for this can be that the rise of stock price may cause enterprises to expand production, which leads to more use of non-clean energy, and less use of clean energy.

Figure 12 summarizes the connectedness between green bonds, clean energy and stock price at different points. There exist obvious differences between three points. During the period of economic downturn, green bonds have greater impacts on stock price. Before the COVID-19 pandemic, stock price may exert positive influence on green bonds, however, stock price only has negative impacts on green bonds during the COVID-19 pandemic. Therefore, the connectedness between three variables is time-varying.

Table 5 displays the comparison of our findings and previous findings. Some findings are consistent with those of previous literature (Hammoudeh et al., 2021; Kung et al., 2022; McInerney \& Bunn, 2019; Urom et al., 2021), but there are also some opposites. For example, we find the positive effect was weakened during the COVID-19 pandemic, contrary to Pham (2021)'s view that the connectedness between green bonds and clean energy can be strengthened during extreme conditions. In addition, we conclude the negative impacts of stock price on clean energy, which is contrary to Al Mamun et al. (2018) and Paramati et al. (2016).

\subsection{Robustness test}

The results' robustness is checked by additional analysis. The robustness can be implemented through a smaller sample (Avkiran \& Cai, 2014; He, 2020). Thus, we use the weekly data spanning from January 06, 2017 to July 09, 2021 to test the robustness. The Markov Chain Monte Carlo algorithm (MCMC) in the context of Bayesian inference simulates 50,000 times, and the result of the first 5000 iterations is a burn-in sample. The results of MCMC estimation is shown in Table 6. The inefficiency factors are less than 100, which indicates that the posterior of the model draws efficiently and are valid for further analysis. Similar to Figs. 3, 13 also shows the simulation process is effective and robust, and the simulation results converge to the posterior distribution.

Figures 14 and 15 report the results of simultaneous relation and impulse response respectively. Similar to Fig. 5, green bonds have an unchanged impact on clean energy. The effects of clean energy on stock price show an increasing trend before 2017, and the trend decreases 


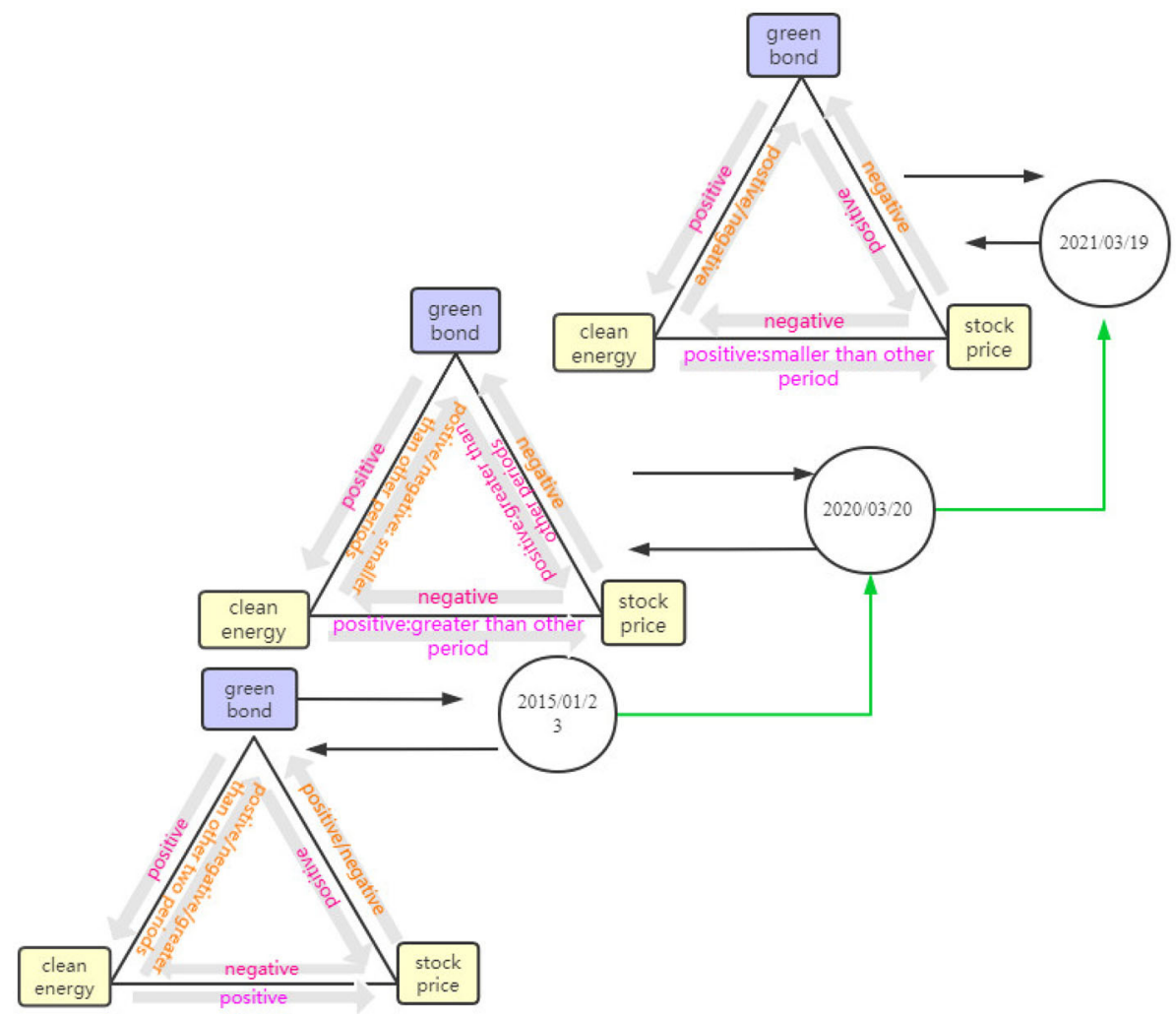

Fig. 12 The relationship between green bonds, clean energy and stock price at different points

after the outbreak of the COVID-19 pandemic. Green bonds also have a decreasing impact on stock price during the COVID-19 pandemic. We use April 28, 2017, March 20, 2020 and March 19, 2021 to represent period before the COVID-19 pandemic, economic downturn period during the COVID-19 and economic recovery period during the COVID-19, respectively. Green bonds can positively impact clean energy and stock price during three periods. And the positive impacts of clean energy on stock price in economic recovery period are greater than in other two periods. The results also indicate that stock price has a negative impact on green bonds and clean energy. Results show the relationship between green bonds, clean energy, and stock price is time-varying, and the results are similar to the results that covering the periods from 2011 to 2021. Therefore, the evidence also indicates that our results are robust.

\section{Conclusion}

Given the important non-linearity, this paper uses the TVP-VAR model to analyze the dynamic non-linear connectedness between the green bonds, clean energy, and stock prices due to the outbreak of the COVID-19 pandemic. Firstly, there is a positive simultaneous relationship between the green bonds, clean energy, and stock prices. However, there are differences 
Table 5 Comparison of our findings with those of previous literature

\begin{tabular}{|c|c|c|}
\hline Variables & Our findings & Previous findings \\
\hline \multirow[t]{2}{*}{ Green bonds $\rightarrow$ clean energy } & $\begin{array}{l}\text { The simultaneous impact of clean } \\
\text { energy to green bonds is positive }\end{array}$ & $\begin{array}{l}\text { The issuance of green bonds can } \\
\text { encourage producers' } \\
\text { willingness to engage with } \\
\text { bioenergy production } \\
\text { (McInerney \& Bunn, 2019) }\end{array}$ \\
\hline & $\begin{array}{l}\text { Green bonds can positively influence } \\
\text { clean energy in the short and long } \\
\text { term }\end{array}$ & $\begin{array}{l}\text { Green bonds suppot the } \\
\text { development of bioenergy } \\
\text { (Kung et al., 2022) }\end{array}$ \\
\hline \multirow[t]{2}{*}{ Clean energy $\rightarrow$ green bonds } & $\begin{array}{l}\text { In the long term, the positive impacts } \\
\text { of clean energy on green bonds are } \\
\text { weak }\end{array}$ & $\begin{array}{l}\text { There exists limited } \\
\text { connectedness between green } \\
\text { bonds and clean energy } \\
\text { (Hammoudeh et al., 2021) }\end{array}$ \\
\hline & $\begin{array}{l}\text { The positive impacts are weakened } \\
\text { during the COVID-19 pandemic }\end{array}$ & $\begin{array}{l}\text { The connectedness between green } \\
\text { bonds and clean energy has been } \\
\text { strengthened during extreme } \\
\text { market conditions (Pham, 2021) }\end{array}$ \\
\hline Green bonds $\rightarrow$ stock price & $\begin{array}{l}\text { Green bonds exert a positive impact } \\
\text { on stock price before and during } \\
\text { the COVID-19 }\end{array}$ & $\begin{array}{l}\text { Green bonds have a positive } \\
\text { impact on stock returns (Wang } \\
\text { et al., 2020) }\end{array}$ \\
\hline Stock price $\rightarrow$ green bonds & $\begin{array}{l}\text { Stock price weakly affects green } \\
\text { bonds }\end{array}$ & $\begin{array}{l}\text { Green bonds are weakly } \\
\text { connected with the stock } \\
\text { (Reboredo \& Ugolini, 2020) }\end{array}$ \\
\hline Clean energy $\rightarrow$ stock price & $\begin{array}{l}\text { Clean energy can positively impact } \\
\text { stock price in the short term }\end{array}$ & $\begin{array}{l}\text { The U.S. and European clean } \\
\text { energy markets can positively } \\
\text { influence global stock returns in } \\
\text { the short term (Urom et al., } \\
\text { 2021) }\end{array}$ \\
\hline Stock price $\rightarrow$ clean energy & $\begin{array}{l}\text { Stock price exerts a negative impact } \\
\text { on clean energy before and during } \\
\text { the COVID-19 }\end{array}$ & $\begin{array}{l}\text { The development of the stock } \\
\text { market can significantly and } \\
\text { positively influence cleaner } \\
\text { energy production (Al Mamun } \\
\text { et al., 2018) } \\
\text { The development of the stock } \\
\text { market exerts a positive impact } \\
\text { on clean energy use (Paramati } \\
\text { et al., 2016) }\end{array}$ \\
\hline
\end{tabular}

Table 6 MCMC estimation results of parameters in robustness test

\begin{tabular}{lllllll}
\hline Parameter & Mean & Std. Dev & $95 \% \mathrm{~L}$ & $95 \% \mathrm{U}$ & Geweke'CD & Inef. \\
\hline$\left(\Sigma_{\beta}\right)_{1}$ & 0.0023 & 0.0003 & 0.0018 & 0.0029 & 0.041 & 12.94 \\
$\left(\Sigma_{\beta}\right)_{2}$ & 0.0023 & 0.0003 & 0.0018 & 0.0028 & 0.161 & 14.96 \\
$\left(\Sigma_{\alpha}\right)_{1}$ & 0.0056 & 0.0016 & 0.0034 & 0.0096 & 0.447 & 73.57 \\
$\left(\Sigma_{\alpha}\right)_{2}$ & 0.0056 & 0.0016 & 0.0034 & 0.0098 & 0.864 & 66.07 \\
$\left(\Sigma_{h}\right)_{1}$ & 0.2174 & 0.0522 & 0.1289 & 0.3333 & 0.953 & 73.08 \\
$\left(\Sigma_{h}\right)_{2}$ & 0.2228 & 0.0479 & 0.1412 & 0.3293 & 0.730 & 58.31 \\
\hline
\end{tabular}



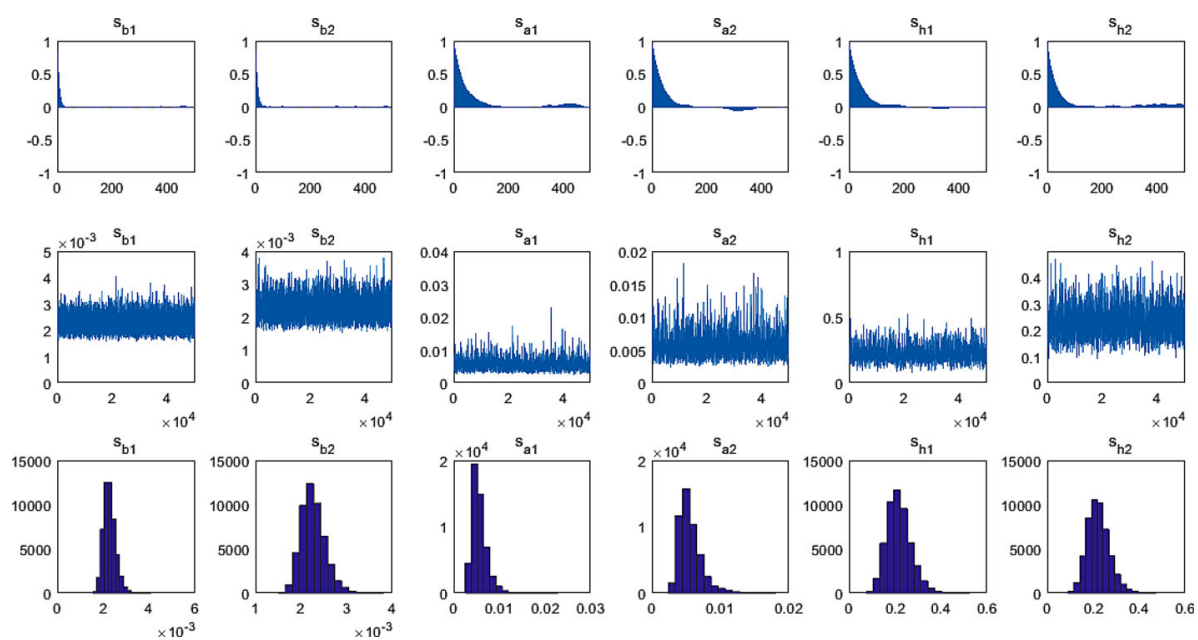

Fig. 13 The autocorrelation coefficient, sample path and posterior distribution in robustness test
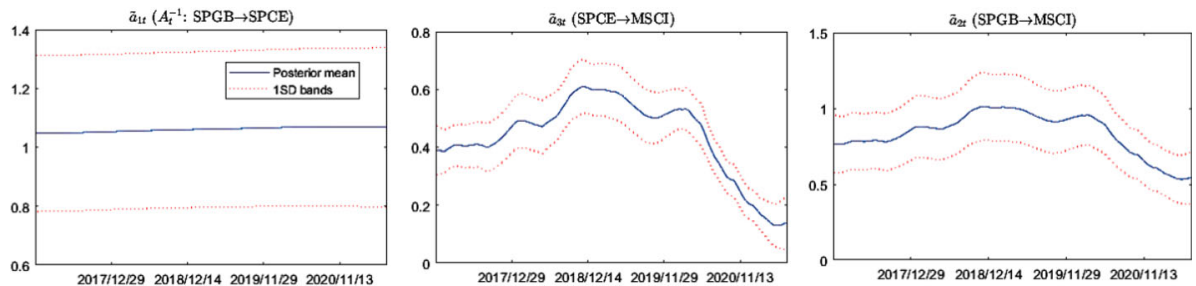

Fig. 14 Simultaneous relation for SPGB, SPCE and MSCI in robustness test
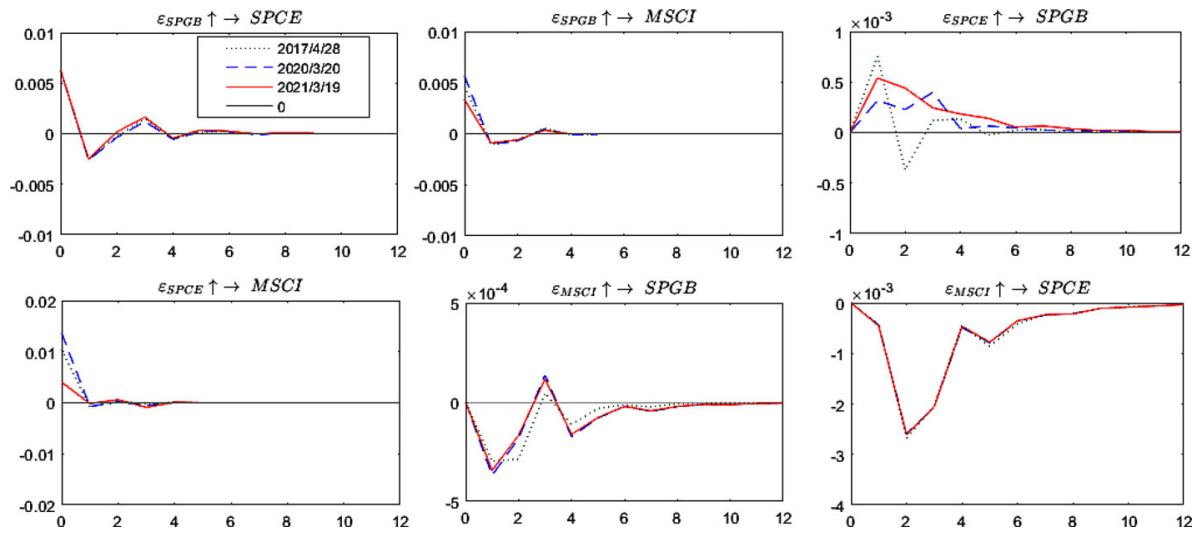

Fig. 15 Time-varying response at different points 
in the positive relationship at different time points. Secondly, in the analysis of impulse response of different time horizons, we find that the green bonds positively impact clean energy in the short term. The positive effects strengthen after the outbreak of the COVID-19 pandemic. The negative effects of the green bonds on stock prices for the medium term in the economic recovery period are weaker than that before the COVID-19 pandemic. In the short term, the development of clean energy has a positive influence on stock prices, and the positive influence increases during the COVID-19 pandemic, indicating that promoting the development of clean energy can promote stock prices and change the downward trend of the global stock market. In the medium, the negative effects of stock prices on green bonds decline during the COVID-19 pandemic. In the short, medium and long term, the improvement of stock prices negatively affects clean energy. Finally, from the perspective of impulse analysis at different points, we conclude that the development of green bonds provides an effective way to finance clean energy which can promote the development of clean energy. During the economic downturn, the development of a green bond market can improve the global economic situation. Therefore, countries around the world should take effective measures to promote the development of green economy to resume production. Moreover, clean energy has a stronger positive impact on stock prices. Compared with the other two points, green bonds have a greater positive impact on the clean energy in the economic recovery period during the COVID-19 pandemic, which can make implications for governments.

Although the development of green economy can resume production, the high cost of green production may lead enterprises to ignore environmental production. Therefore, government should be committed to reducing the financing costs of issuing green bonds to encourage the issuance of green bonds during the COVID-19 pandemic. Moreover, the transmission effect of incentive policies should be further improved, and capital investment should be guided to green industries. It is recommended to further explore the establishment of a full-market and full-variety green bond investment section to provide convenience for investors to track market performance and choose green bonds. In addition, legislative and institutional guarantees should be made as important suggestions. During the COVID-19 pandemic period, stock prices have a greater negative impact on the clean energy. This can be attributed to companies increasing production to compensate for the losses caused by the COVID-19 pandemic, while ignoring environmental benefits. Therefore, the government should strengthen supervision and actively adopt legislative measures to require companies to strictly disclose environmental information. Internet technologies, such as big data and blockchain, can realize information sharing between various departments and prevent enterprises from neglecting environmental benefits for their own development.

According to the conclusion, this paper puts forward some policy recommendations from the three perspectives of the government, financial regulatory authorities, enterprises and investors. First of all, the government should attach great importance to green development and accelerate the energy transition. The government can consider measures such as green subsidies or tax relief for environmentally friendly companies to achieve this goal. Second, the financial regulatory authorities of all countries should take active measures to establish and improve the green financial system. In this way, financial institutions are guided to vigorously develop green financial products and increase investment in clean energy. Third, enterprises should upgrade their industrial structure to ultimately achieve sustainable economic development. For this purpose, enterprises can make full use of the government's green subsidies and green credit policies of the financial institutions mentioned in the previous proposal to actively invest funds in technological transformation and promptly eliminate backward industries that cannot transform. In this way, the upgrading of industrial structure brings about green economic growth. Moreover, investors and enterprises should improve 
their risk management capabilities to respond to extreme events. In the long term, green bonds weakly connect with clean energy and stock price, it's thereby recommended to combine green bonds and stock markets in the portfolio to obtain diversified benefits. Moreover, since the dependence is time-varying, enterprises and investors should focus on dynamic characteristics while making investment decisions.

Our results have several useful implications for investors, policymakers and enterprises. From the perspective of portfolio diversification, our results have strong implications for the investors. Our results suggest investors combine green bonds with clean energy and stock assets to obtain diversified benefits. Our results also indicate that the connectedness between different markets are time-varying. Therefore, investors should pay more attention to extreme market conditions to take reasonable risk management measures. Our findings are also beneficial for policymakers. Countries around the world are take measures to recover economy damaged by the COVID-19 pandemic. Green development can be an effective way to develop economy and achieve environmental commitment. As an environmentally friendly financial instruments, green bonds can raise funds for green industries, green projects or green economic activities. Hence, it's imperative to make policies to promote the development of green bonds. We suggest reducing the costs of issuing green bonds, improving the information disclosure system and standardizing the green bond market to accelerate the development of green bond markets. Our findings indicate green bonds play a crucial role in clean energy markets, which contribute to energy transition. Additionally, enterprises can use green financial tools, such as green bond, to raise fund to resume production.

Although our study has investigated the connectedness between green bonds, clean energy and stock price, there exists differences in economic development and policies across different countries and regions. Therefore, future research should be directed to exclusively discuss the connectedness between green bonds, clean energy and stock price in specific country or region. And targeted suggestions can be put forward according to national and regional conditions.

Acknowledgements This work was supported by the National Natural Science Foundation of China (Nos. 71704098, 71971039, 72001191, 72003110), and the Natural Science Foundation of Henan Province (No. 202300410442).

\section{References}

Abu-Rayash, A., \& Dincer, I. (2020). Analysis of the electricity demand trends amidst the COVID-19 coronavirus pandemic. Energy Research \& Social Science, 68, 101682.

Adekoya, O. B., \& Oliyide, J. A. (2021). How COVID-19 drives connectedness among commodity and financial markets: evidence from TVP-VAR and causality-in-quantiles techniques. Resources Policy, 70, 101898.

Al Mamun, M., Sohag, K., Shahbaz, M., \& Hammoudeh, S. (2018). Financial markets, innovations and cleaner energy production in OECD countries. Energy Economics, 72, 236-254.

Ali Shah, S. A., Longsheng, C., Solangi, Y. A., Ahmad, M., \& Ali, S. (2021). Energy trilemma based prioritization of waste-to-energy technologies: implications for post-COVID-19 green economic recovery in Pakistan. Journal of Cleaner Production, 284, 124729.

Ashraf, B. N. (2021). Stock markets' reaction to Covid-19: Moderating role of national culture. Finance Research Letters, 41, 101857.

Avkiran, N. K., \& Cai, L. (2014). Identifying distress among banks prior to a major crisis using non-oriented super-SBM. Annals of Operations Research, 217(1), 31-53.

Azomahou, T. T., Ndung, U. N., \& Ouédraogo. (2021). Coping with a dual shock: The economic effects of COVID-19 and oil price crises on African economies. Resources Policy, 72, 102093.

Baker, S. R., Bloom, N., Davis, S. J., Kost, K., Sammon, M., \& Viratyosi, T. (2020). The unprecedented stock market impact of COVID-19. Working Paper 26945, National Bureau of Economic Research. 
Baumeister, C., \& Peersman, G. (2013). Time-varying effects of oil supply shocks on the US economy. American Economic Journal: Macroeconomics, 5(4), 1-28.

Benedetti, D., Biffis, E., Chatzimichalakis, F., Fedele, L. L., \& Simm, I. (2021). Climate change investment risk: Optimal portfolio construction ahead of the transition to a lower-carbon economy. Annals of Operations Research, 299(1), 847-871.

BloombergNEF. (2020). Power sector to spend \$5 billion on software by 2025. Retrieved from https://about. bnef.com/blog/power-sector-to-spend-5-billion-on-software-by-2025/.

Borjigin, S., Yang, Y., Yang, X., \& Sun, L. (2018). Econometric testing on linear and nonlinear dynamic relation between stock prices and macroeconomy in China. Physica A: Statistical Mechanics and Its Applications, 493, 107-115.

Campiglio, E. (2016). Beyond carbon pricing: The role of banking and monetary policy in financing the transition to a low-carbon economy. Ecological Economics, 121, 220-230.

Chatziantoniou, I., Filippidis, M., Filis, G., \& Gabauer, D. (2021). A closer look into the global determinants of oil price volatility. Energy Economics, 95, 105092.

Chen, C., Zarazua De Rubens, G., Xu, X., \& Li, J. (2020). Coronavirus comes home? Energy use, home energy management, and the social-psychological factors of COVID-19. Energy Research \& Social Science, 68, 101688 .

European Commission. (2016). Study on the potential of green bond finance for resource efficient investments. Retrieved from. http://ec.europa.eu/environment/enveco/pdf/potential-greenbond.pdf.

Dawar, I., Dutta, A., Bouri, E., \& Saeed, T. (2021). Crude oil prices and clean energy stock indices: Lagged and asymmetric effects with quantile regression. Renewable Energy, 163, 288-299.

Dincer, I., \& Acar, C. (2015). A review on clean energy solutions for better sustainability. International Journal of Energy Research, 39(5), 585-606.

Elsayed, A. H., Nasreen, S., \& Tiwari, A. K. (2020). Time-varying co-movements between energy market and global financial markets: implication for portfolio diversification and hedging strategies. Energy Economics, 90, 104847.

Esmaeili, P., \& Rafei, M. (2021). Dynamics analysis of factors affecting electricity consumption fluctuations based on economic conditions: Application of SVAR and TVP-VAR models. Energy, 226, 120340.

Ferrer, R., Shahzad, S. J. H., \& Soriano, P. (2021). Are green bonds a different asset class? Evidence from time-frequency connectedness analysis. Journal of Cleaner Production, 292, 125988.

Forster, P. M., Forster, H. I., Evans, M. J., Gidden, M. J., Jones, C. D., Keller, C. A., \& Turnock, S. T. (2020). Current and future global climate impacts resulting from COVID-19. Nature Climate Change, 10(10), 913-919.

Ftiti, Z., Ben Ameur, H., \& Louhichi, W. (2021). Does non-fundamental news related to COVID-19 matter for stock returns? Evidence from Shanghai stock market. Economic Modelling, 99, 105484.

Fuller, D. W. A. (1979). Distribution of the estimators for autoregressive time series with a unit root. Journal of the American Statistical Association, 79(366), 355-367.

Gao, Y., Li, Y., \& Wang, Y. (2021). Risk spillover and network connectedness analysis of China's green bond and financial markets: Evidence from financial events of 2015-2020. The North American Journal of Economics and Finance, 57, 101386.

Geng, J., Chen, F., Ji, Q., \& Liu, B. (2021). Network connectedness between natural gas markets, uncertainty and stock markets. Energy Economics, 95, 105001.

Gong, X., Shi, R., Xu, J., \& Lin, B. (2021). Analyzing spillover effects between carbon and fossil energy markets from a time-varying perspective. Applied Energy, 285, 116384.

Granger, C. (2008). Non-linear models: Where do we go next - time varying parameter models? Studies in Nonlinear Dynamics \& Econometrics, 12(3), 1639.

Gu, X., Zhu, Z., \& Yu, M. (2021). The macro effects of GPR and EPU indexes over the global oil market-are the two types of uncertainty shock alike? Energy Economics, 100, 105394.

Halbrügge, S., Schott, P., Weibelzahl, M., Buhl, H. U., Fridgen, G., \& Schöpf, M. (2021). How did the German and other European electricity systems react to the COVID-19 pandemic? Applied Energy, 285, 116370.

Hammoudeh, S., Ajmi, A. N., \& Mokni, K. (2021). Relationship between green bonds and financial and environmental variables: A novel time-varying causality. Energy Economics, 92, 104941.

He, Z. (2020). Dynamic impacts of crude oil price on Chinese investor sentiment: Nonlinear causality and time-varying effect. International Review of Economics and Finance, 66, 131-153.

He, L., Zhang, L., Zhong, Z., Wang, D., \& Wang, F. (2019). Green credit, renewable energy investment and green economy development: Empirical analysis based on 150 listed companies of China. Journal of Cleaner Production, 208, 363-372.

Huang, S., \& Liu, H. (2021). Impact of COVID-19 on stock price crash risk: Evidence from Chinese energy firms. Energy Economics, 101, 105431. 
Huang, Y., Duan, K., \& Mishra, T. (2021). Is Bitcoin really more than a diversifier? A pre- and post-COVID-19 analysis. Finance Research Letters, 43, 102016.

Huang, T., \& Yue, Q. (2020). How the game changer was generated? An analysis on the legal rules and development of china's green bond market. International Environmental Agreements: Politics, Law and Economics, 20(1), 85-102.

Initiative, C.B. (2019). 2019 Green Bond Market Summary. Retrieved from. https://www.climatebonds.net/ resources/reports/2019-green-bond-market-summary.

IRENA (International Renewable Energy Agency) (2020). Post-COVID recovery: An agenda forresilience, development and equality. Retrieved from. https://www.irena.org/publications/20 20/Jun/Post-COVIDRecovery.

Kassouri, Y., Kacou, K. Y. T., \& Alola, A. A. (2021). Are oil-clean energy and high technology stock prices in the same straits? Bubbles speculation and time-varying perspectives. Energy, 232, 121021.

Kuang, W. (2021). Are clean energy assets a safe haven for international equity markets? Journal of Cleaner Production, 302, 127006.

Kumah, F. Y. (2007). A markov-switching approach to measuring exchange market pressure. IMF Working Papers, 07(242), 1.

Kung, C., Lan, X., Yang, Y., Kung, S., \& Chang, M. (2022). Effects of green bonds on Taiwan's bioenergy development. Energy, 238, 121567.

Le Quéré, C., Peters, G. P., Friedlingstein, P., Andrew, R. M., Canadell, J. G., Davis, S. J., \& Jones, M. W. (2021). Fossil $\mathrm{CO}_{2}$ emissions in the post-COVID-19 era. Nature Climate Change, 11(3), 197-199.

Lei, X., Shiyun, T., Yanfei, D., \& Yuan, Y. (2020). Sustainable operation-oriented investment risk evaluation and optimization for renewable energy project: A case study of wind power in China. Annals of Operations Research, 290(1), 223-241.

Li, F., Cao, X., \& Ou, R. (2021). A network-based evolutionary analysis of the diffusion of cleaner energy substitution in enterprises: the roles of PEST factors. Energy Policy, 156, 112385.

Lin, L., Zhou, Z., Liu, Q., \& Jiang, Y. (2019). Risk transmission between natural gas market and stock markets: Portfolio and hedging strategy analysis. Finance Research Letters, 29, 245-254.

Liu, X., Wang, Z., Sun, X., Zhang, L., \& Zhang, M. (2020). Clarifying the relationship among clean energy consumption, haze pollution and economic growth-based on the empirical analysis of China's Yangtze River Delta Region. Ecological Complexity, 44, 100871.

Liu, Y., Wei, Y., Wang, Q., \& Liu, Y. (2021). International stock market risk contagion during the COVID-19 pandemic. Finance Research Letters. https://doi.org/10.1016/j.frl.2021.102145

Liu, N., Liu, C., Da, B., Zhang, T., \& Guan, F. (2021). Dependence and risk spillovers between green bonds and clean energy markets. Journal of Cleaner Production, 279, 123595.

Liu, X., \& Zeng, M. (2017). Renewable energy investment risk evaluation model based on system dynamics. Renewable and Sustainable Energy Reviews, 73, 782-788.

Loiseau, E., Saikku, L., Antikainen, R., Droste, N., Hansjuergens, B., Pitkanen, K., \& Thomsen, M. (2016). Green economy and related concepts: An overview. Journal of Cleaner Production, 139, 361-371.

Lundgren, A. I., Milicevic, A., Uddin, G. S., \& Kang, S. H. (2018). Connectedness network and dependence structure mechanism in green investments. Energy Economics, 72, 145-153.

McInerney, C., \& Bunn, D. W. (2019). Expansion of the investor base for the energy transition. Energy Policy, $129,1240-1244$.

Mensi, W., Vo, X. V., \& Kang, S. H. (2021). Upside-downside multifractality and efficiency of green bonds: the roles of global factors and COVID-19. Finance Research Letters, 43, 101995.

Monasterolo, I., \& Raberto, M. (2018). The EIRIN flow-of-funds behavioural model of green fiscal policies and green sovereign bonds. Ecological Economics, 144, 228-243.

Naeem, M. A., Farid, S., Ferrer, R., \& Shahzad, S. J. H. (2021). Comparative efficiency of green and conventional bonds pre- and during COVID-19: An asymmetric multifractal detrended fluctuation analysis. Energy Policy, 153, 112285.

Nakajima, J. (2011). Time-varying parameter VAR model with stochastic volatility: An overview of methodology and empirical applications. Monetary and Economic Studies, 29, 107-142.

Nguyen, T. T. H., Naeem, M. A., Balli, F., Balli, H. O., \& Vo, X. V. (2021). Time-frequency co-movement among green bonds, stocks, commodities, clean energy, and conventional bonds. Finance Research Letters, 40, 101739.

Nundy, S., Ghosh, A., Mesloub, A., Albaqawy, G. A., \& Alnaim, M. M. (2021). Impact of COVID-19 pandemic on socio-economic, energy-environment and transport sector globally and sustainable development goal (SDG). Journal of Cleaner Production, 312, 127705.

Paramati, S. R., Ummalla, M., \& Apergis, N. (2016). The effect of foreign direct investment and stock market growth on clean energy use across a panel of emerging market economies. Energy Economics, 56, $29-41$. 
Pham, L. (2021). Frequency connectedness and cross-quantile dependence between green bond and green equity markets. Energy Economics, 98, 105257.

Phillips, P. C. B., \& Perron, P. (1988). Testing for a unit root in time series regression. Biometrika, 75(2), 335-346.

Piotr, F., \& Witold, O. (2018). Nonlinear granger causality between grains and livestock. Agricultural Economics, 64(7), 328-336.

Primiceri, G. E. (2005). Time varying structural vector autoregressions and monetary policy. Review of Economic Studies, 3, 821-852.

Razmi, S. F., Bajgiran, B. R., Behname, M., Salari, T. E., \& Razmi, S. M. J. (2020). The relationship of renewable energy consumption to stock market development and economic growth in Iran. Renewable Energy, 145, 2019-2024.

Reboredo, J. C. (2018). Green bond and financial markets: Co-movement, diversification and price spillover effects. Energy Economics, 74, 38-50.

Reboredo, J. C., \& Ugolini, A. (2020). Price connectedness between green bond and financial markets. Economic Modelling, 88, 25-38.

Sari, R., Ewing, B. T., \& Soytas, U. (2008). The relationship between disaggregate energy consumption and industrial production in the United States: An ARDL approach. Energy Economics, 30(5), 2302-2313.

Strantzali, E., \& Aravossis, K. (2016). Decision making in renewable energy investments: A review. Renewable and Sustainable Energy Reviews, 55, 885-898.

Tisdell, C. A. (2020). Economic, social and political issues raised by the COVID-19 pandemic. Economic Analysis and Policy, 68, 17-28.

Uddin, G. S., Rahman, M. L., Hedström, A., \& Ahmed, A. (2019). Cross-quantilogram-based correlation and dependence between renewable energy stock and other asset classes. Energy Economics, 80, 743-759.

UN (United Nations). (2021). World Economic Situation and Prospects as of mid-2021. Retrieved from. https://www.un.org/development/desa/dpad/publication/world-economic-situation-and-prospects-asof-mid-2021/

UNEP (United Nations Environment Programme). (2010). Green economy reports: a preview; UNEP: Nairobi, Kenya.

Urom, C., Abid, I., Guesmi, K., \& Chevallier, J. (2020). Quantile spillovers and dependence between Bitcoin, equities and strategic commodities. Economic Modelling, 93, 230-258.

Urom, C., Mzoughi, H., Abid, I., \& Brahim, M. (2021). Green markets integration in different time scales: a regional analysis. Energy Economics, 98, 105254.

Verschuur, J., Koks, E. E., \& Hall, J. W. (2021). Observed impacts of the COVID-19 pandemic on global trade. Nature Human Behaviour, 5(3), 305-307.

Wan, D., Xue, R., Linnenluecke, M., Tian, J., \& Shan, Y. (2021). The impact of investor attention during COVID-19 on investment in clean energy versus fossil fuel firms. Finance Research Letters, 43, 101955.

Wang, J., Chen, X., Li, X., Yu, J., \& Zhong, R. (2020). The market reaction to green bond issuance: Evidence from China. Pacific-Basin Finance Journal, 60, 101294.

Wu, J., Huang, D., Zhou, Z., \& Zhu, Q. (2020b). The regional green growth and sustainable development of China in the presence of sustainable resources recovered from pollutions. Annals of Operations Research, 290(1), 27-45.

Wu, Y., Wang, J., Ji, S., \& Song, Z. (2020). Renewable energy investment risk assessment for nations along China's Belt \& Road Initiative: An ANP-cloud model method. Energy, 190, 116381.

$\mathrm{Xu}$, L. (2021). Stock return and the COVID-19 pandemic: Evidence from Canada and the US. Finance Research Letters, 38, 101872.

Yi, X., Bai, C., Lyu, S., \& Dai, L. (2021). The impacts of the COVID-19 pandemic on China's green bond market. Finance Research Letters, 42, 101948.

Yildirim, E. (2014). Energy use, $\mathrm{CO}_{2}$ emission and foreign direct investment: Is there any inconsistence between causal relations? Frontiers in Energy, 8(3), 269-278.

Zhang, H., Cai, G., \& Yang, D. (2020). The impact of oil price shocks on clean energy stocks: Fresh evidence from multi-scale perspective. Energy, 196, 117099.

Zhou, M., Huang, J., \& Chen, J. (2020). The effects of geopolitical risks on the stock dynamics of China's rare metals: A TVP-VAR analysis. Resources Policy, 68, 101784.

Publisher's Note Springer Nature remains neutral with regard to jurisdictional claims in published maps and institutional affiliations. 